IMA Journal of Applied Mathematics (2016) Page 1 of 39

doi:10.1093/imamat/iarxxx

\title{
Dynamics of a slowly evaporating solvent-polymer with a deformable upper surface
}

\author{
MatThew G. HenNeSSY* AND ANDREAS MÜNCH \\ Mathematical Institute, University of Oxford, Woodstock Road, Oxford, UK, OX2 6GG \\ Corresponding author: hennessy@maths.ox.ac.uk
}

[Received on XX XX XXXX; revised on XX XX XXXX; accepted on XX XX XXXX]

\begin{abstract}
This paper examines how surface deformations affect the stability of a slowly evaporating solventpolymer mixture. The destabilising effect of surface-tension variations arising from evaporation-induced concentration gradients and the counteracting influence of mean gravity and surface tension are incorporated into the mathematical model. A linear stability analysis that takes advantage of the separation between the characteristic time scales of the slowly evolving base state and the perturbations is carried out in combination with numerical solutions of the linearised system. It is shown that the onset of instability can occur for Marangoni numbers that are much lower than the critical value for a non-deformable surface. Moreover, two types of Marangoni instabilities appear in the system: one is associated with the traditional stationary instability, and the other is an oscillatory instability that is not present for a nondeformable liquid surface. A region of the parameter space where the oscillatory instability dominates is identified and used to formulate appropriate conditions for future experiments.
\end{abstract}

Keywords: oscillatory Marangoni instability; evaporating films; multiple scales; WKB approximation.

\section{Introduction}

Evaporating solvent-polymer mixtures play an important role in a wide variety of industrial processes such as painting, inkjet printing, and the fabrication of polymer-based photovoltaic devices; see Howison et al. (1997); de Gans et al. (2004); Heriot \& Jones (2005); Günes et al. (2007); Souche \& Clarke (2009). As these mixtures dry they are influenced by several factors including, but not limited to, surface tension, surface-tension gradients, gravity, and van der Waals interactions. In the case when multiple polymers are blended in a solvent, the change in temperature and composition that occurs during the drying process can trigger the onset of phase separation within the bulk (Moons, 2002). All of these factors influence the dynamics of the evaporating film and as a result, they can each have a profound impact on the resulting polymer morphology. For instance, surface tension is known to flatten the surface of films, whereas van der Waals forces can lead to the rupture of thin films. For a review on the dynamics of thin layers of fluid, see Myers (1998); Oron et al. (1997); Craster \& Matar (2009). Different industrial applications seek different polymer morphologies; for example, the polymers that constitute the photoactive component of organic photovoltaic devices should interpenetrate on nanometer scales (Moons, 2002). Therefore, understanding the relationship between these various physical factors and the morphology of evaporating solvent-polymer films is of great practical importance.

Evaporating solvent-polymer mixtures that are assumed to be isothermal are, in many ways, analogous to films of a pure liquid that are heated from below. More specifically, evaporation of solvent from the upper surface can induce a concentration gradient within the layer which mimics the temperature gradient in a fluid on top of a heated substrate. In the case when the solvent and the polymer have 
different densities, a concentration gradient can lead to buoyancy effects in much the same way that the thermal expansion of a pure liquid can. Furthermore, composition and temperature can affect the surface tension of a fluid in the same manner. The close analogy between the two systems suggests that the vast literature on pure liquid films heated from below can provide useful insight into the dynamics of evaporating solvent-polymer blends.

The first systematic studies of a pure liquid layer subject to heating from below are the early experiments by Bénard (1900, 1901a,b), who showed that certain experimental conditions can lead to the onset of convection within the layer. Rayleigh (1916) subsequently performed a mathematical analysis of Bénard's experiments and found that convection could be driven by thermally-induced density variations within the fluid layer. However, Block (1956) later showed that Bénard's films were too thin for convection to be driven by density differences, and instead proposed that convection was caused by thermally-induced gradients in the surface tension. Block's hypothesis was later verified in a mathematical analysis by Pearson (1958). Scriven \& Sternling (1964) extended Pearson's work to the case when the fluid layer has a deformable surface, but their prediction that long-wavelength convection should be observed in films with arbitrarily small Marangoni numbers contradicted several experimental results; see Koschmieder (1993). The discrepancy between experiment and theory was reconciled by Smith (1966), who showed that gravity stabilises long-wavelength convection. Further experiments by Berg et al. (1966) highlighted how evaporation can lead to the onset of thermal convection in fluid layers that are not heated from below. In these systems the temperature gradient is caused by evaporative cooling at the surface of the fluid. An extensive theoretical investigation of evaporating layers of pure fluid has been carried out by Burelbach et al. (1988). Sultan et al. (2005) extended this work by considering the diffusion of vapour in the overlying gas. For recent developments in the field of convective instabilities in liquid films, see Bodenschatz et al. (2000) and Colinet et al. (2001).

In layers of pure fluid that are subject to a vertical temperature gradient, the instability that is driven by surface-tension gradients, i.e., the Marangoni instability, can either be stationary or oscillatory in nature. Stationary instabilities are associated with the monotonic growth or amplification of small disturbances, whereas oscillatory instabilities lead to disturbances that grow in a modulated fashion. In the case of fluid layers with non-deformable upper surfaces, numerical studies by Vidal \& Acrivos (1966) suggested the Marangoni instability had to be stationary, and this was later proven by Vrentas \& Vrentas (2004). However, when the surface of the fluid is deformable, Takashima (1981) showed that an oscillatory Marangoni instability is possible if the bottom of the fluid layer is cooler than the top. Subsequent studies by Pérez-García \& Carneiro (1991) and Shklyaev et al. (2012) found that this was also possible in systems that are heated from below. Shklyaev et al. (2010) has shown the existence of thermallydriven long-wavelength oscillatory instabilities in layers of pure fluid when the temperature gradient is induced by evaporation at the free surface.

Given the close connection between layers of pure fluid that are subject to a vertical temperature gradient and evaporating isothermal solvent-solute mixtures, it is natural to wonder if the oscillatory Marangoni instability can occur in the latter system as well. The experiments with waterborne coatings by Kojima et al. (1995) might support such a claim. During the drying process the crests of the initial surface deformation can become troughs, and troughs can become crests. This crest-to-trough reversal has been reported to happen several times during a given experiment, and it could represent a standing wave solution of the governing equations. This reversal has also been found in drying paint layers, although usually only one reversal occurs during a given experiment; see Overdiep (1986), Howison et al. (1997), or Wilson (1993) for more details. Further mathematical investigations of the reversal have been carried out by Eres et al. (1999) in the framework of lubrication theory and under the assumption that the solute concentration is uniform across the height of the fluid layer. Their frozen-mode anal- 
ysis indicates that, for sufficiently large evaporation rates, oscillatory modes can be linearly unstable. However, their nonlinear simulations show that the system is stabilised due to the viscosity of the mixture increasing as solvent is removed and hence the oscillations decay. In this paper we will carry out a systematic linear stability analysis for the governing equations that includes the effects of long-time changes to the base state via a method developed in Hennessy \& Münch (2013) and extended to dewetting rims in Dziwnik et al. (2014). Our formulation of the problem does not depend on the lubrication assumption, hence the analysis captures both long-wave oscillatory and short-wave stationary modes and their competition. Particular attention is paid to identifying regions in parameter space where the dominant mode of instability is oscillatory. Since gravity is known to stabilise the long-wavelength stationary instability in films with deformable surfaces, we will include it in our model to determine whether it affects the presence of unstable long-wavelength oscillatory modes as well.

The mathematical analysis of evaporating layers of fluid is complicated by the fact that the evaporative mass loss naturally leads to solutions that depend on time. In the context of linear stability theory, this often implies that the base state which is perturbed about is time dependent. As a result, the linearised problem for the perturbations becomes non-autonomous in the time variable which generally means that the solution will no longer simply be proportional to $\exp (\lambda t)$. In some instances, such as in Burelbach et al. (1988), this is not an issue and the linear stability problem can still be solved analytically. In other cases the stability problem can be simplified by seeking a transformation that separates the space and time variables or by seeking a similarity solution in certain time regimes; see Smolka \& Witelski (2009) and Kang \& Choi (1997). As mentioned by Davis (1976) in the context of hydrodynamic stability, Floquet theory can be used when the base state is periodic in time.

In the situation where the linear stability problem cannot be treated analytically, one must turn to an approximate method or make some simplifying assumptions about the base state. Machrafi et al. (2011) treat the stability problem as an initial value problem which is then solved numerically. Doumenc et al. (2010) describe how to find the optimal initial condition that maximises the perturbation amplitude at a given time, and they show that their numerical results are in good agreement with experiment. However, numerical approaches can quickly become prohibitive if the parameter space of the problem is large, and in this case it becomes highly desirable to have a systematic method for determining the stability of the system over a wide range of parameter values.

A common approach for simplifying a non-autonomous stability problem is the so-called frozentime method, which is based around the assumption that the base state evolves so slowly that it can be frozen. That is, the time dependence of the base state is dropped and it is treated as an additional system parameter. This reduces the stability problem to an autonomous one and it has the advantage of allowing the usual techniques from linear stability theory to be applied. However, this approach only provides information about the instantaneous stability of the problem for a given base state and therefore, it is difficult to assess how the stability of the problem changes over the long times on which the base state evolves. Despite this shortcoming, the frozen-time method has been used in a number of studies of evaporating mixtures, for example, Machrafi et al. (2010); Haut \& Colinet (2005); Eres et al. (1999), and in a related work without gravity by Serpetsi \& Yiantsios (2012).

The long-term stability of the problem can be assessed via numerical simulation, but as Hennessy \& Münch (2013) point out, several authors have noticed a close connection between the eigenvalues of the frozen-time linear stability problem and the approximate solutions that are obtained by solving the nonautonomous stability problem numerically. In particular, Lick (1965) used a WKB-like approach for the case of time-varying heating to show that the solution to the linearised problem can be approximated by integrating the largest frozen-time eigenvalue in time and then taking the exponential. A corresponding result and the next-order correction were computed for the evaporation-driven Marangoni instability by 
Hennessy and Münch using the method of multiple scales. This approach is built around the separation of time scales that occurs when the base state evolves much slower than perturbations to it. Using their asymptotic solution, Hennessy and Münch were able to track the slow changes in the stability that occur as a binary mixture with a non-deformable surface slowly evaporates.

This paper considers the evolution of an evaporating layer of fluid with a deformable interface that is composed of a single non-volatile polymer and a volatile solvent. In addition to evaporation influencing the fluid dynamics of the mixture, we include the action of gravity, surface tension, and surface-tension gradients in the mathematical model. The strength of these effects are characterised by a Galileo number, a capillary number, and a Marangoni number, respectively. We neglect the effects of buoyancy and we assume the fluid is isothermal. The latter assumption is motivated by the findings of Bassou \& Rharbi (2009), who show that thermal effects are likely to be small in evaporating toluene-polystyrene blends. Recent work by Chauvet et al. (2012) has also shown that evaporation can dampen thermocapillary effects. The precise formulation of the mathematical model is given in Section 2. We use this model to investigate possible modes of instability in evaporating solvent-polymer systems with deformable surfaces and therefore, we analyse the system using linear stability theory. The base state is time dependent and thus the regular stability techniques do not directly apply. However, once the base state settles into a quasi-equilibrium it evolves on the evaporative time scale whereas perturbations to it evolve on the diffusive time scale. Therefore, by assuming that evaporation is slow compared to diffusion, these time scales separate and the quasi-steady base state evolves much slower than perturbations to it. This enables us to invoke the frozen-time method to deduce how the instantaneous stability of the base state varies with the three non-dimensional numbers mentioned above. With this information we identify parameter regimes for which the frozen-time analysis predicts an instability, and we use an extension of the asymptotic solution presented in Hennessy \& Münch (2013) to determine the maximum amplification of disturbances to the base state in the unstable regimes. Our analysis indicates that surface deformations affect the stability in two ways compared to when the surface is non-deformable: the onset of instability can occur at much lower Marangoni numbers and the instability can be oscillatory in nature. Details of the mathematical analysis are given in Section 3 and two derivations of the asymptotic solution to the linear stability problem are presented in Appendix A. One derivation is based on the method of multiple scales and the other uses the WKB approximation. The results of the linear stability analysis using the frozen-time and asymptotic methods are given in Section 4 . We interpret the results in an experimental context in Section 5 and we provide experimental conditions that could lead to the appearance of an oscillatory instability. A summary and conclusions follow in Section 6.

\section{Mathematical model}

We are interested in the dynamics of an evaporating layer of isothermal fluid that is composed of a volatile solvent and a non-volatile polymer. The fluid layer is assumed to be of infinite horizontal extent and it rests on a non-permeable substrate; see Figure 1. Above the fluid layer we assume the existence of a passive gas layer; that is, we neglect any interactions between this gas layer and the underlying fluid. Neglecting this gas layer can be made systematic by taking the so-called one-sided limit of a two-layer (fluid and gas) model; see Burelbach et al. (1988) for more details. The mathematical model therefore considers the horizontal and vertical components of the mixture velocity, $u$ and $w$, respectively; the hydrodynamic pressure $p$; and the volume fraction of solvent $c$; all of which are functions of the horizontal coordinate $x$, the vertical coordinate $z$, and time $t$. 


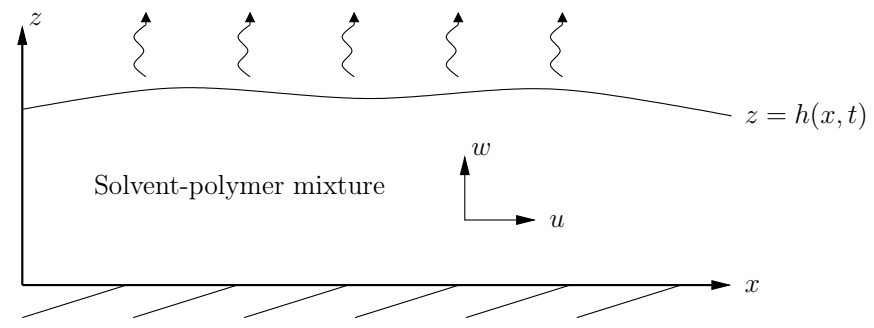

FIG. 1. A schematic diagram of the system under consideration, showing a two-dimensional layer of fluid resting on a nonpermeable substrate. The fluid is composed of a non-volatile polymer and a volatile solvent. The layer has thickness $h(x, t)$ and the mixture has horizontal and vertical components of velocity given by $u$ and $w$, respectively.

Conservation of solvent and polymer within the fluid layer implies

$$
\begin{aligned}
\partial_{t} \rho_{s}+\nabla \cdot \mathbf{q}_{s} & =0, \\
\partial_{t} \rho_{p}+\nabla \cdot \mathbf{q}_{p} & =0,
\end{aligned}
$$

where $\rho_{s}$ and $\rho_{p}$ denote the density of the solvent and polymer, respectively, and $\mathbf{q}_{s}$ and $\mathbf{q}_{p}$ denote the corresponding mass fluxes. We assume the density of the mixture $\rho=\rho_{s}+\rho_{p}$ is constant and we define the mixture flux as $\mathbf{q}=\rho \mathbf{u}=\mathbf{q}_{s}+\mathbf{q}_{p}$, where $\mathbf{u}=u \mathbf{e}_{x}+w \mathbf{e}_{z}$ is the velocity written in terms of the basis vectors $\mathbf{e}_{x}$ and $\mathbf{e}_{z}$ pointing in the $x$ and $z$ direction, respectively. Adding the two equations in (2.1) implies the mixture velocity is divergence free, thus

$$
\nabla \cdot \mathbf{u}=0
$$

The solvent mass flux is assumed to have two contributions: one from the bulk motion of the mixture and the other from diffusion into the polymer. Therefore, we take $\mathbf{q}_{s}=\rho_{s} \mathbf{u}-D \nabla \rho_{s}$, where $D$ is the diffusion coefficient of solvent into polymer. By writing the solvent density in terms of the solvent volume fraction, $\rho_{s}=\rho c$, the conservation equation in (2.1a) becomes

$$
\partial_{t} c+\mathbf{u} \cdot \nabla c=D \nabla^{2} c
$$

where (2.2) has been used and the diffusion coefficient $D$ has been assumed constant. The bulk motion of the mixture is assumed to be governed by the Stokes equations,

$$
-\nabla p+\mu \nabla^{2} \mathbf{u}-\rho g \mathbf{e}_{z}=0,
$$

where $\mu$ denotes the kinematic viscosity of the mixture (assumed constant), and $g$ is the acceleration due to gravity.

The assumption that the mixture is Newtonian with constant viscosity and diffusivity is only expected to be valid during the early stages of the drying process when the polymer phase is dilute. For later times, the nonlinear dependence of the viscosity and diffusivity on the polymer concentration and nonNewtonian behaviour due to shear thinning are expected to influence the dynamics of the evaporating mixture. If the initial polymer concentration is sufficiently dilute, however, the mixture will behave Newtonian during the first stages of the drying process and, in particular, when the instability is first developing. Thus, a Newtonian fluid model with constant viscosity is a useful approximation that can be used to gain basic insight into role of surface deformations and to explore the presence of oscillatory 
instabilities in an evaporating mixture. We therefore neglect these complicated rheological features for the time being but will discuss them further in the conclusion.

The underlying substrate at $z=0$ is assumed to be non-permeable and therefore, the usual no-flux and no-slip conditions apply,

$$
u=w=\partial_{z} c=0 .
$$

The surface of the fluid layer is denoted by $z=h(x, t)$ and it is assumed that only the solvent evaporates. A rigorous mathematical description of the evaporation process cannot be obtained from our one-sided model because it neglects the effects of the overlying gas layer. Instead, we take a phenomenological approach and assume the evaporative mass flux is proportional to the solvent concentration at the surface,

$$
q_{\text {evap }}=k_{m} \rho c,
$$

where $k_{m}$ is a mass transfer coefficient. Such relations are common in studies involving evaporating solvent-solute systems; see Bornside et al. (1989) and Souche \& Clarke (2009).

Conservation of total mass across the surface of the fluid layer implies that

$$
\rho\left(\mathbf{u} \cdot \mathbf{e}_{n}-v_{n}\right)=q_{\text {evap }},
$$

where $\mathbf{e}_{n}$ and $v_{n}$ are the unit normal vector to the surface and the normal velocity of the surface, respectively. These can be written explicitly as

$$
\mathbf{e}_{n}=\frac{-\partial_{x} h \mathbf{e}_{x}+\mathbf{e}_{z}}{\sqrt{1+\left(\partial_{x} h\right)^{2}}}, \quad v_{n}=\frac{\partial_{t} h}{\sqrt{1+\left(\partial_{x} h\right)^{2}}} .
$$

Conservation of solvent across the surface yields the condition

$$
-\rho D \nabla c \cdot \mathbf{e}_{n}+\rho c\left(\mathbf{u} \cdot \mathbf{e}_{n}-v_{n}\right)=q_{\mathrm{evap}} .
$$

By combining (2.7) and (2.9), a simplified mass-balance condition for the solvent can be obtained, namely

$$
-\rho D \nabla c \cdot \mathbf{e}_{n}=q_{\text {evap }}(1-c) .
$$

Deformations in the surface of the fluid layer and gradients in the surface tension both induce stresses that must be balanced by the pressure and the viscous stress in the fluid at the surface. The normal and tangential stress balances at the interface are given by

$$
\begin{aligned}
-p+\mu \mathbf{e}_{n} \cdot\left(\nabla \mathbf{u}+\nabla \mathbf{u}^{T}\right) \cdot \mathbf{e}_{n} & =-\gamma \nabla \cdot \mathbf{e}_{n}, \\
\mu \mathbf{e}_{t} \cdot\left(\nabla \mathbf{u}+\nabla \mathbf{u}^{T}\right) \cdot \mathbf{e}_{n} & =\partial_{s} \gamma,
\end{aligned}
$$

respectively, where $\mathbf{e}_{t}$ denotes the unit tangent vector to the surface; $\partial_{s}$ denotes differentiation with respect to the arclength of the surface; and $\gamma$ is the surface tension of the fluid, which we take to be a linearly decreasing function of the solvent concentration at the surface, $\gamma=\gamma_{r}-\gamma_{c} \rho\left(c-c_{r}\right)$. Here, $\gamma_{c}$ and $c_{r}$ are reference values and $\gamma_{c}$ characterises how the surface tension varies with the solvent volume fraction. The unit tangent vector is given by

$$
\mathbf{e}_{t}=\frac{\mathbf{e}_{x}+\partial_{x} h \mathbf{e}_{z}}{\sqrt{1+\left(\partial_{x} h\right)^{2}}}
$$


Using the expression for the surface tension in (2.11b) yields the boundary condition

$$
\mu \mathbf{e}_{t} \cdot\left(\nabla \mathbf{u}+\nabla \mathbf{u}^{T}\right) \cdot \mathbf{e}_{n}=-\gamma_{c} \rho \nabla c \cdot \mathbf{e}_{t},
$$

The initial conditions for the solvent volume fraction and the film thickness are given by

$$
c(x, z, 0)=c_{i}(x, z), \quad h(x, 0)=h_{i}(x) .
$$

We define the mean values of the initial conditions as

$$
\beta=\lim _{L \rightarrow \infty} \frac{1}{2 L} \int_{-L}^{L} \frac{1}{h_{i}(x)} \int_{0}^{h_{i}(x)} c_{i}(x, z) \mathrm{d} z \mathrm{~d} x, \quad h_{m}=\lim _{L \rightarrow \infty} \frac{1}{2 L} \int_{-L}^{L} h_{i}(x) \mathrm{d} x,
$$

and these will be used in the non-dimensionalisation below.

\section{$2.1 \quad$ Non-dimensionalisation}

We scale the spatial dimensions of the problem with the mean initial thickness of the film so that $x=h_{m} x^{\prime}$ and $z=h_{m} z^{\prime}$, where primes are used to denote dimensionless quantities. Time is scaled according to the diffusive time scale, $t=\left(h_{m}^{2} / D\right) t^{\prime}$, and from these we can define a velocity scale so that $\mathbf{u}=\left(D / h_{m}\right) \mathbf{u}^{\prime}$. The pressure scale is chosen to be $\mu D / h_{m}^{2}$ and we write the pressure as the deviation from its hydrostatic component, $p=\left(\mu D / h_{m}^{2}\right)\left(p^{\prime}-\mathrm{Ga} z^{\prime}\right)$, where $\mathrm{Ga}$ is the Galileo number defined as

$$
\mathrm{Ga}=\frac{\rho g h_{m}^{3}}{\mu D} .
$$

The solvent volume fraction is written as the deviation from its mean initial value, $c=\beta+S c^{\prime}$, where the scale $S$ is determined from the boundary condition (2.10), where $c$ is replaced by $\beta$ on the right-hand side of this condition. This gives $S=\delta \beta(1-\beta)$, where

$$
\delta=\frac{k_{m} h_{m}}{D}
$$

defines an evaporative Biot number.

With these scalings the bulk equations can be written as (after dropping the primes)

$$
\begin{aligned}
-\nabla p+\nabla^{2} \mathbf{u} & =0, \\
\nabla \cdot \mathbf{u} & =0, \\
\partial_{t} c+\mathbf{u} \cdot \nabla c & =\nabla^{2} c .
\end{aligned}
$$

The boundary conditions at $z=0$ are given by

$$
u=w=\partial_{z} c=0,
$$

and at the free surface $z=h(x, t)$ they are

$$
\begin{aligned}
& \mathbf{u} \cdot \mathbf{e}_{n}-v_{n}=\delta \beta[1+\delta(1-\beta) c], \\
& -\nabla c \cdot \mathbf{e}_{n}=[1+\delta(1-\beta) c](1-\delta \beta c), \\
& -p+\mathbf{e}_{n} \cdot\left(\nabla \mathbf{u}+\nabla \mathbf{u}^{T}\right) \cdot \mathbf{e}_{n}=-\mathrm{Ga} h-\mathrm{Ca}^{-1}(1-\mathrm{MaCa} c) \nabla \cdot \mathbf{e}_{n}, \\
& \mathbf{e}_{t} \cdot\left(\nabla \mathbf{u}+\nabla \mathbf{u}^{T}\right) \cdot \mathbf{e}_{n}=-\operatorname{Ma} \nabla c \cdot \mathbf{e}_{t},
\end{aligned}
$$


TABLE 1. Example parameter values taken from the experiments of Bassou \& Rharbi (2009)

\begin{tabular}{ccc}
\hline Quantity & Value & Unit \\
\hline$\rho$ & 1000 & $\mathrm{~kg} \cdot \mathrm{m}^{-3}$ \\
$\mu$ & 0.15 & $\mathrm{~Pa} \cdot \mathrm{s}$ \\
$g$ & 9.81 & $\mathrm{~m} \cdot \mathrm{s}^{-2}$ \\
$D$ & $1.38 \times 10^{-9}$ & $\mathrm{~m}^{2} \cdot \mathrm{s}^{-1}$ \\
$k_{m}$ & $3.53 \times 10^{-7}$ & $\mathrm{~m} \cdot \mathrm{s}$ \\
$\gamma_{r}$ & 0.03 & $\mathrm{~N} \cdot \mathrm{m}^{-1}$ \\
$\gamma_{c}$ & $9.2 \times 10^{-6}$ & $\mathrm{~N} \cdot \mathrm{m}^{2} \cdot \mathrm{kg}^{-1}$ \\
$h_{m}$ & $10^{-4}-1.4 \times 10^{-3}$ & $\mathrm{~m}$ \\
$\beta$ & 0.85 & - \\
\hline
\end{tabular}

where we have chosen $c_{r}=\beta$. The non-dimensional initial conditions are given by

$$
c(x, z, 0)=c_{i}(x, z), \quad h(x, 0)=h_{i}(x)
$$

where $c_{i}$ now has a mean value of zero. For a flat and spatially uniform (well-mixed) initial state, the initial conditions are $c_{i}(x, z) \equiv 0$ and $h_{i}(x) \equiv 1$. The additional non-dimensional numbers that appear are the capillary number $\mathrm{Ca}$ and the Marangoni number $\mathrm{Ma}$, and these are defined as

$$
\mathrm{Ca}=\frac{\mu D}{\gamma_{r} h_{m}}, \quad \mathrm{Ma}=\frac{\delta \beta(1-\beta) \rho \gamma_{c} h_{m}}{\mu D} .
$$

In this paper we assume that $\gamma_{c}>0$ so that the Marangoni numbers are positive. However, it is possible for binary mixtures to have negative Marangoni numbers if the solute has the effect the lowering the surface tension of the fluid.

To estimate the size of the non-dimensional numbers, we base the parameters values on the evaporating toluene-polystyrene systems studied by Bassou \& Rharbi (2009). Their mixtures often had an initial solvent volume fraction of $\beta=0.85$ and they imposed an evaporative flux of $q_{\text {evap }}=3 \times 10^{-4}$ $\mathrm{kg} \cdot \mathrm{m}^{-2} \cdot \mathrm{s}^{-1}$. With these two parameter values the mass transfer coefficient $k_{m}$ can be inferred via (2.6) if $c$ is replaced by $\beta$. The density of polystyrene and toluene are $1050 \mathrm{~kg} \cdot \mathrm{m}^{-3}$ and $867 \mathrm{~kg} \cdot \mathrm{m}^{-3}$, respectively, so we approximate the net density of the mixture as $\rho=1000 \mathrm{~kg} \cdot \mathrm{m}^{-3}$. A diffusion coefficient for their system can be obtained by interpolating the values found in NaNagara et al. (1992). A list of the physical parameters in our model, along with their values, can be found in Table 1.

By inserting these parameter values into the expressions for the non-dimensional numbers, we find that $\delta$, which can be interpreted as the ratio of the diffusive time scale to the evaporative time scale $h_{m} / k_{m}$, is bounded between $0.026<\delta<0.36$. This suggests a separation of time scales in the problem and we will assume throughout the remainder of this paper that $\delta \ll 1$. The Galileo and Marangoni numbers can vary by several orders of magnitude owing to their nonlinear dependence on the mean initial film thickness. We find that $14<\mathrm{Ma}<3 \times 10^{3}$ and $47<\mathrm{Ga}<1.3 \times 10^{5}$. The capillary numbers always remain small, however, and these are bounded by $5 \times 10^{-6}<\mathrm{Ca}<7 \times 10^{-5}$. The product $\mathrm{MaCa}$ is always expected to be small so we drop these terms from the normal stress balance $(2.18 \mathrm{~g})$. Moreover, we only consider the case $\mathrm{Ca} \ll 1$ in the calculations below. 


\section{Analysis}

The goal of this paper is to understand how small disturbances to a horizontally-uniform base state evolve in time. Disturbances that grow indicate the possibility of an instability in the system. With this in mind we seek solutions to (2.18) of the form

$$
\begin{aligned}
\mathbf{u}(x, z, t) & =\overline{\mathbf{u}}(z, t)+\varepsilon \tilde{\mathbf{u}}(z, t) e^{i k x}, \\
p(x, z, t) & =\bar{p}(z, t)+\varepsilon \tilde{p}(z, t) e^{i k x}, \\
c(x, z, t) & =\bar{c}(z, t)+\varepsilon \tilde{c}(z, t) e^{i k x}, \\
h(x, t) & =\bar{h}(t)+\varepsilon \tilde{h}(t) e^{i k x},
\end{aligned}
$$

where $\varepsilon \ll 1$ is a small parameter representing the initial size of the disturbance. The $O(1)$ contribution to this solution is called the base state and the associated variables are denoted with bars. The $O(\varepsilon)$ contribution is the disturbance and this is denoted by variables with tildes.

We insert (3.1) into (2.18) and linearise around small $\varepsilon$, thus producing a nonlinear $O(1)$ problem corresponding to the evolution of the base state, and a linear $O(\varepsilon)$ problem that describes the evolution of the disturbances. This formulation assumes that the disturbances remain small enough compared to the base state for the linearisation to be valid. Thus, we expect our analysis to break down when the disturbances become so large that nonlinear effects become important.

\subsection{Computation of the base state}

The base state is determined from the $O(1)$ problem, and we find that the fluid problem corresponds to a hydrostatic situation with solution $\overline{\mathbf{u}}=0, \bar{p}=\mathrm{Ga} \bar{h}(t)$. The $O(1)$ solvent volume fraction satisfies a diffusion equation

$$
\partial_{t} \bar{c}=\partial_{z z} \bar{c}
$$

with boundary conditions

$$
\begin{array}{ll}
\partial_{z} \bar{c}=0, & z=0, \\
\partial_{z} \bar{c}=-[1+\delta(1-\beta) \bar{c}](1-\delta \beta \bar{c}), & z=\bar{h}(t) .
\end{array}
$$

We assume that the initial base state solvent volume fraction corresponds to a well-mixed state, so that $\bar{c}(z, 0) \equiv 0$.

The free boundary evolves according to

$$
\partial_{t} \bar{h}=-\delta \beta[1+\delta(1-\beta) \bar{c}(\bar{h}(t), t)],
$$

and the initial condition for the base state film thickness is $\bar{h}(0)=1$.

An analysis of (3.2) shows that there are two time regimes in this problem. The first occurs when $t=O(1)$, whereas the second occurs when $t$ is large, in particular, when $t=O\left(\delta^{-1}\right)$. During the first time regime evaporation leads to the formation of a solutal boundary layer at the upper surface of the film. This boundary layer grows in thickness until it reaches the substrate, after which the concentration profile begins to settle into a quasi-equilibrium. Up to this point the mass loss due to evaporation has not been large enough to cause a significant decrease in the film thickness. The second time regime describes the quasi-static evolution of the solvent and the thinning of the fluid layer. 
For very small times it is possible to obtain a closed-form expression for the growing boundary layer. We suppose that $t \ll 1$ and we define a fast time given by $T=\delta^{-q} t$, where $q>0$. On this time scale the film thickness remains constant to leading order, thus $\bar{h}(t) \equiv 1$. We expect a boundary layer in the solvent volume fraction at the free surface so we decompose the solution into two parts: an outer solution valid near the substrate and in the bulk, and an inner solution valid near the surface. The outer solution is given trivially by $\bar{c}(z, t) \equiv 0$, and to find the inner solution we first scale the solvent volume fraction as $c=\delta^{q / 2} \varphi$ and let $z=1-\delta^{q / 2} \eta$. The leading-order problem for $\varphi$ is given by

$$
\partial_{T} \varphi=\partial_{\eta \eta} \varphi
$$

subject to $\partial_{\eta} \varphi=1$ at $\eta=0, \varphi \rightarrow 0$ as $\eta \rightarrow \infty$, and $\varphi=0$ at $T=0$. The solution to this problem can be found using a Laplace transform and the uniformly-valid composite solution is given by

$$
\bar{c}(z, t)=(1-z) \operatorname{erfc}\left(\frac{1-z}{\sqrt{4 t}}\right)-\sqrt{\frac{4 t}{\pi}} e^{-(1-z)^{2} / 4 t}, \quad t \ll 1 .
$$

The solutions for larger times are discussed in detail by Hennessy \& Münch (2013), so we only give an overview of the results here. When $t=O(1)$ the solution can be found using a Fourier series and it is given by

$$
\begin{aligned}
\bar{c}(z, t) & =\frac{1}{6}-\frac{1}{2} z^{2}-t+\frac{2}{\pi^{2}} \sum_{n=1}^{\infty} \frac{(-1)^{n}}{n^{2}} e^{-n^{2} \pi^{2} t} \cos (n \pi z)+O(\delta), \\
\bar{h}(t) & =1+O(\delta) .
\end{aligned}
$$

The boundary layer solution in (3.4) is, in fact, contained within this solution, so that (3.5) provides a complete description of the solution in the first time regime.

The solution in the second time regime is most conveniently written in terms of the slow time $\tau=\delta t$. The solvent volume fraction is given by

$$
\bar{c}(z, \tau)=\delta^{-1}\left(\frac{\bar{h}(\tau)-1}{\beta \bar{h}(\tau)}\right)-\frac{1}{2} F(\tau) z^{2}+E(\tau)+O(\delta),
$$

where $E$ is an unspecified function of the slow time (it is not needed in the later problems) that satisfies $E(0)=1 / 6$. The function $F$, which is measure of the gradient in the solvent concentration, is given by

$$
F(\tau)=\frac{\bar{h}(\tau)-1+\beta}{\beta \bar{h}^{3}(\tau)} .
$$

The leading-order film thickness is given implicitly by

$$
\bar{h}-1+(1-\beta) \log \left(\frac{\bar{h}-1+\beta}{\beta}\right)=-\tau .
$$

The asymptotic solutions to the base state are compared to numerical solutions in Figure 2 for the example parameter values $\beta=0.85$ and $\delta=0.01$. Although this hypothetical value of $\delta$ is slightly smaller than the experimental values from Bassou \& Rharbi (2009), we find that increasing $\delta$ does not affect the qualitative features of the dynamics. The numerical scheme is based on a method-of-lines approach where spatial derivatives are discretised using finite differences and the resulting equations 


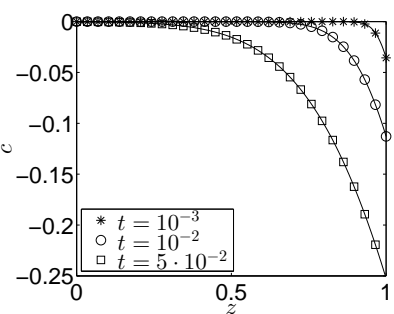

(a)

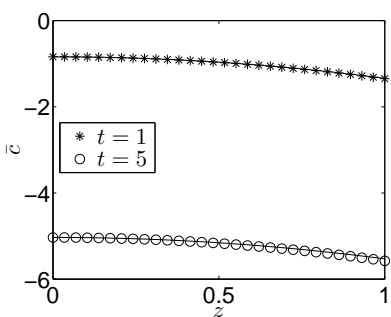

(b)

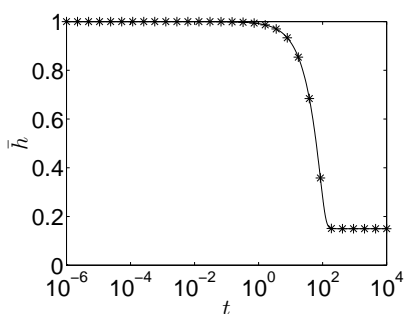

(c)

FIG. 2. Numerical (solid lines) and asymptotic (symbols) solutions to the base state problem. (a): The short-term behaviour of the base state solvent volume fraction showing, in particular, the growth of a boundary layer from the surface of the fluid layer. (b): The quasi-static evolution of the solvent volume fraction. (c): The evolution of the film thickness. The asymptotic solution in (3.4) is used in panel (a), whereas the $t=O\left(\delta^{-1}\right)$ asymptotic solution given in (3.6) is used in panels (b)-(c). Parameter values are $\beta=0.85$ and $\delta=0.01$.

are treated as a system of ordinary differential equations (ODEs). Figure 2 (a) shows the asymptotic solution for small times given in (3.4) and it highlights the fast growth of a solutal boundary layer from the free upper surface. The quasi-steady solution to the solvent volume fraction (3.6a) is presented in Figure 2 (b), and it can be seen that the concentration profiles differ mainly by an additive constant. The evolution of the film thickness and a comparison of the quasi-steady solution (3.6c) is shown in Figure 2 (c). From the figure it can be seen that the film thickness remains relatively constant up up to $O(1)$ times, after which it slowly decreases to its equilibrium value of $1-\beta$.

Throughout the remainder of the paper we neglect the fast initial transients in the base state and we consider only its quasi-steady evolution. That is, we assume that the base state solution is given by (3.6) for all time. As Figure 2 shows, this solution captures much of the base state evolution, even for $O(1)$ times, so this is not too unrealistic of an assumption. Moreover, the slow evolution of the base state can be exploited to simplify the $O(\varepsilon)$ problem for the perturbations and in particular, it will allow the problem to be solved using asymptotic methods for slowly-varying problems.

\subsection{Linear stability}

The $O(\varepsilon)$ problem describes the linear stability of the base state. The perturbation to the horizontal velocity $\tilde{u}$ can be eliminated from this problem via the $O(\varepsilon)$ incompressibility condition which reads $i k \tilde{u}+\partial_{z} \tilde{w}=0$. The remaining bulk equations for this problem can be written as

$$
\begin{aligned}
-k^{2} \tilde{p}+\partial_{z z} \tilde{p} & =0, \\
-\partial_{z} \tilde{p}-k^{2} \tilde{w}+\partial_{z z} \tilde{w} & =0, \\
\partial_{t} \tilde{c}+\tilde{w} \partial_{z} \bar{c} & =-k^{2} \tilde{c}+\partial_{z z} \tilde{c},
\end{aligned}
$$

and these are subject to the boundary conditions

$$
\tilde{w}=\partial_{z} \tilde{w}=\partial_{z} \tilde{c}=0
$$


at $z=0$. The corresponding boundary conditions at the free surface are linearised about the base state film thickness and therefore, at $z=\bar{h}(\tau)$, we impose

$$
\begin{aligned}
\tilde{w}-\partial_{t} \tilde{h} & =\delta^{2} \beta(1-\beta)\left[\tilde{c}+\left(\partial_{z} \bar{c}\right) \tilde{h}\right], \\
\left(\partial_{z z} \tilde{c}\right) \tilde{h}+\partial_{z} \tilde{c} & =-\delta[1-2 \beta-2 \delta \beta(1-\beta) \bar{c}]\left[\tilde{c}+\left(\partial_{z} \bar{c}\right) \tilde{h}\right], \\
-\tilde{p}+2 \partial_{z} \tilde{w} & =-\left(k^{2} \mathrm{Ca}^{-1}+\mathrm{Ga}\right) \tilde{h}, \\
\partial_{z z} \tilde{w}+k^{2} \tilde{w} & =-k^{2} \operatorname{Ma}\left[\tilde{c}+\left(\partial_{z} \bar{c}\right) \tilde{h}\right] .
\end{aligned}
$$

The pressure disturbance $\tilde{p}$ can also be eliminated from this problem by combining (3.7a) and (3.7b) to yield $\tilde{p}=k^{-2} \partial_{z z z} \tilde{w}-\partial_{z} \tilde{w}$. This implies that (3.7b) can be written as

$$
\left(\partial_{z z}-k^{2}\right)^{2} \tilde{w}=0
$$

and the boundary condition $(3.7 \mathrm{~g})$ becomes

$$
-\partial_{z z z} \tilde{w}+3 k^{2} \partial_{z} \tilde{w}=-k^{2}\left(k^{2} \mathrm{Ca}^{-1}+\mathrm{Ga}\right) \tilde{h} .
$$

Initial conditions for the problem are given by

$$
\tilde{c}(z, 0)=\tilde{c}_{i}(z), \quad \tilde{h}(0)=\tilde{h}_{i} .
$$

Throughout the remainder of the paper we neglect terms of $O(\delta)$ and higher that appear in the linear stability problem (3.7) and in particular, in its boundary conditions, which is asymptotically consistent with using a solution for the base state that is $O(1)$ accurate.

3.2.1 Frozen-time analysis In the frozen-time analysis we treat the slow time $\tau$ as a parameter and not as a time variable. The interpretation of this approximation is that if the base state evolves slow enough then it looks approximately constant. This can be made asymptotically rigorous by treating $\tau$ as a time variable, taking the limit $\delta \rightarrow 0$ with $t$ fixed and $\tau=\delta t$, and then considering the leadingorder problem. Under this assumption the linear problem given in (3.7) becomes autonomous and the dynamics can be deduced from an eigenvalue analysis. Thus, we seek solutions of the form

$$
\begin{aligned}
\tilde{w}(z, t) & =\hat{w}(z ; \tau) e^{\lambda(\tau) t}, \\
\tilde{c}(z, t) & =\hat{c}(z ; \tau) e^{\lambda(\tau) t}, \\
\tilde{h}(t) & =\hat{h}(\tau) e^{\lambda(\tau) t},
\end{aligned}
$$

where $\lambda$ is an eigenvalue corresponding to the instantaneous growth rate of the disturbances at time $\tau$. Inserting (3.11) into (3.7), using (3.2) for the base state, and neglecting the $O(\delta)$ terms yields the eigenvalue problem given by

$$
\begin{aligned}
\left(\partial_{z z}-k^{2}\right)^{2} \hat{w} & =0, \\
\lambda \hat{c}-F z \hat{w} & =-k^{2} \hat{c}+\partial_{z z} \hat{c},
\end{aligned}
$$

where the $\tau$ dependence of $\lambda$ and $F$ has not been written for brevity. Boundary conditions for this problem are

$$
\hat{w}=\partial_{z} \hat{w}=\partial_{z} \hat{c}=0
$$


along the substrate $z=0$ and

$$
\begin{aligned}
\hat{w}-\lambda \hat{h} & =0, \\
-F \hat{h}+\partial_{z} \hat{c} & =0 \\
-\partial_{z z z} \hat{w}+3 k^{2} \partial_{z} \hat{w} & =-k^{2}\left(k^{2} \mathrm{Ca}^{-1}+\mathrm{Ga}\right) \hat{h}, \\
\partial_{z z} \hat{w}+k^{2} \hat{w} & =-\operatorname{Ma} k^{2}(\hat{c}-F \bar{h} \hat{h}),
\end{aligned}
$$

at the linearised free surface $z=\bar{h}(\tau)$.

To simplify the eigenvalue problem in (3.12), we rescale the variables and parameters in a manner similar to Hennessy \& Münch (2013) by letting

$$
\begin{aligned}
& z=\bar{h}(\tau) \zeta, \quad k=\bar{h}^{-1}(\tau) a, \quad \lambda=\bar{h}^{-2}(\tau) \sigma, \\
& \hat{w}=F^{-1}(\tau) \bar{h}^{-3}(\tau) \check{w}, \quad \hat{c}=\check{c}, \quad \hat{h}=F^{-1}(\tau) \bar{h}^{-1}(\tau) \check{h} .
\end{aligned}
$$

The corresponding rescaled eigenvalue problem is given by

$$
\begin{aligned}
\left(\partial_{\zeta \zeta}-a^{2}\right)^{2} \check{w} & =0, \\
\sigma \check{c}-\zeta \check{w} & =-a^{2} \check{c}+\partial_{\zeta \zeta} \check{c} .
\end{aligned}
$$

The boundary conditions at $\zeta=0$ read

$$
\check{w}=\partial_{\zeta} \check{w}=\partial_{\zeta} \check{c}=0
$$

and at $\zeta=1$ we have

$$
\begin{aligned}
& \check{w}-\sigma \breve{h}=0, \\
& -\check{h}+\partial_{\zeta} \check{c}=0,
\end{aligned}
$$

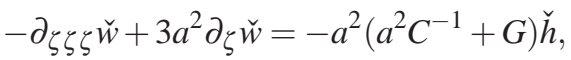

$$
\begin{aligned}
& \partial_{\zeta \zeta} \check{w}+a^{2} \check{w}=-a^{2} M(\check{c}-\check{h}) .
\end{aligned}
$$

The numbers $M, C$, and $G$ that appear in the boundary conditions denote effective, time-dependent non-dimensional numbers given by

$$
M=F(\tau) \bar{h}(\tau) \mathrm{Ma}, \quad C=\bar{h}^{-1}(\tau) \mathrm{Ca}, \quad G=\bar{h}^{3}(\tau) \mathrm{Ga} .
$$

When $\tau=0$ these numbers are equivalent to the original non-dimensional parameters $\mathrm{Ma}$, $\mathrm{Ca}$, and $\mathrm{Ga}$.

The $\tau$ dependence now enters this problem through the rescaled wavenumber $a$ and the effective non-dimensional numbers $G, C$, and $M$. Thus, understanding how the stability of the base state evolves under changes in $\tau$ is equivalent to understanding how the eigenvalue $\sigma$ changes with $a, G, C$, and $M$. From the rescaling in (3.15) and the definition of $F$ in (3.6b) we have that the effective Marangoni number $M$ monotonically decreases to zero as $\bar{h} \rightarrow 1-\beta$. Thus, the destabilising Marangoni stresses weaken as the system evolves and we expect that the system will eventually stabilise.

An examination of (3.14) shows that the problem for the vertical velocity $\check{w}$ decouples from the solvent problem and thus it can be solved to find

$$
\check{w}(\zeta)=\check{W}_{1}(\zeta) \check{c}(1)+\check{W}_{2}(\zeta) \check{h},
$$


where $\breve{W}_{1}$ and $\breve{W}_{2}$ are complicated functions of $\zeta$, the parameters, and the wavenumber $a$. This solution can be inserted into the bulk equation for the solvent volume fraction (3.14b) which can then be solved to obtain

$$
\check{c}(\zeta)=C_{1} \cosh (\alpha \zeta)+C_{2} \sinh (\alpha \zeta)+\int_{0}^{1} \xi \check{w}(\xi) \Phi(\xi ; \zeta) \mathrm{d} \xi
$$

where $C_{1}$ and $C_{2}$ are constants of integration, $\alpha^{2}=\sigma+a^{2}$ is related to the eigenvalue, and $\Phi$ is a Green's function defined as

$$
\Phi(\xi ; \zeta)= \begin{cases}\frac{\cosh (\alpha(\zeta-1)) \cosh (\alpha \xi)}{\alpha \sinh (\alpha)}, & 0<\xi<\zeta \\ \frac{\cosh (\alpha(\xi-1)) \cosh (\alpha \zeta)}{\alpha \sinh (\alpha)}, & \zeta<\xi<1\end{cases}
$$

By inserting the solutions for $\check{w}$ and $\check{c}$ into the boundary conditions $(3.14 \mathrm{c}),(3.14 \mathrm{~d})$, and $(3.14 \mathrm{e})$, and by evaluating (3.17) at $\zeta=1$, a linear system can be obtained for the four remaining unknowns $C_{1}, C_{2}$, $\breve{h}$, and $\check{c}(1)$. Setting the determinant of this linear system equal to zero provides an additional relation that can be used to compute the eigenvalues $\sigma$, and in practice this highly nonlinear equation is solved numerically.

3.2.2 Asymptotic analysis If the base state is "unfrozen" so that $\tau$ is treated properly as a time variable, then it is possible to construct an asymptotic solution to the non-autonomous linear stability problem (3.7) using the method of multiple scales (Kevorkian \& Cole, 1996) or using the WKB approximation (Bender \& Orszag, 1999). These solutions will be uniformly valid over long periods of time. Both cases begin by first eliminating $\tilde{w}$ so that (3.7) (without its $O(\delta)$ and higher contributions) reduces to an evolution problem of the form

$$
\partial_{t} \tilde{U}=L(\tau) \tilde{U}
$$

where $L$ is a slowly varying linear operator and $\tilde{U}(z, t)=[\tilde{c}(z, t), \tilde{h}(t)]^{T}$. We write the boundary conditions as $B(\tau) \tilde{U}=0$, where $B$ denotes a linear boundary operator, and the initial conditions as $\tilde{U}(z, 0)=$ $\tilde{U}_{i}(z)=\left[\tilde{c}_{i}(z), \tilde{h}_{i}\right]^{T}$.

The two different asymptotic treatments of this problem are given in Appendix A so the details will not be presented here. The results of the two methods are the same, and in both cases the solution $U$ is written as an eigenfunction expansion of the form

$$
\tilde{U}(z, t)=\sum_{l=1}^{\infty} \alpha_{l}(t) v_{l}(z ; \tau)
$$

where $v_{l}$ denotes the $l$-th eigenfunction which satisfies

$$
L(\tau) v_{l}=\lambda_{l}(\tau) v_{l}
$$

with $B(\tau) v_{l}=0$ on the boundary. The normalisation of these eigenfunctions will be discussed below and the eigenvalues are labelled according to the size of their real part; $\operatorname{Re}\left\{\lambda_{1}\right\}>\operatorname{Re}\left\{\lambda_{2}\right\}>\ldots>\operatorname{Re}\left\{\lambda_{n}\right\}$ as $n \rightarrow \infty$. Note that the eigenvalues and eigenfunctions of (3.21) exactly correspond to those of the original frozen-time problem (3.12). 
The asymptotic expression for the coefficients $\alpha_{l}$ in (3.20) are given by

$$
\begin{aligned}
\alpha_{l}(t)=\alpha_{l i} e^{T_{l}(t)} & +\delta \sum_{j \neq l}^{\infty} \frac{\alpha_{j i} \gamma_{l j}(\tau)}{\lambda_{j}(\tau)-\lambda_{l}(\tau)} e^{T_{j}(t)} \\
& +\delta \sum_{j \neq l}^{\infty}\left[\alpha_{l i} \int_{0}^{\tau} \frac{\gamma_{l j}(s) \gamma_{j l}(s)}{\lambda_{l}(s)-\lambda_{j}(s)} \mathrm{d} s-\frac{\alpha_{j i} \gamma_{l j}(0)}{\lambda_{j}(0)-\lambda_{l}(0)}\right] e^{T_{l}(t)}+O\left(\delta^{2}\right),
\end{aligned}
$$

where the $T_{l}$ represent additional fast and slow times defined as

$$
T_{l}(t)=\delta^{-1} \int_{0}^{\delta t}\left[\lambda_{l}(s)+\delta \gamma_{l l}(s)\right] \mathrm{d} s, \quad l=1,2, \ldots
$$

Furthermore, $\alpha_{l i}$ denotes the initial value of $\alpha_{l}$, defined as $\alpha_{i}=\left\langle v_{l}^{*}, \tilde{U}_{i}\right\rangle^{\dagger}(0)$, and the $\gamma_{j l}$ are related to the slow-time derivatives of the eigenfunctions,

$$
\gamma_{j l}(\tau)=-\left\langle v_{j}^{*}, \partial_{\tau} v_{l}\right\rangle^{\dagger}(\tau)
$$

Here the dagger denotes complex conjugation, $v_{l}^{*}$ is the $l$-th adjoint eigenfunction, and $\langle\cdot, \cdot\rangle$ is the $\tau$ dependent inner product which is defined for two functions $\hat{U}_{j}=\left[\hat{c}_{j}(z), \hat{h}_{j}\right]^{T}, j=1,2$, as

$$
\left\langle\hat{U}_{1}, \hat{U}_{2}\right\rangle(\tau)=\int_{0}^{\bar{h}(\tau)} \hat{c}_{1}(z) \hat{c}_{2}^{\dagger}(z) \mathrm{d} z+F^{2}(\tau) \bar{h}^{3}(\tau) \hat{h}_{1} \hat{h}_{2}^{\dagger} .
$$

This unusual choice of inner product is made because it allows the rescaled frozen-time eigenfunctions in (3.14) to be used in the calculation of inner products instead of the original eigenfunctions in (3.12). This can be seen by transforming the functions $\hat{U}_{1}$ and $\hat{U}_{2}$ in (3.25) into two new functions, $\breve{U}_{j}=$ $\left[\check{c}_{j}(\zeta), \breve{h}_{j}\right]^{T}, j=1,2$, using the rescaling in (3.13). The inner product in (3.25) can then be written as

$$
\left\langle\hat{U}_{1}, \hat{U}_{2}\right\rangle(\tau)=\bar{h}(\tau)\left[\int_{0}^{1} \check{c}_{1}(\zeta) \check{c}_{2}^{\dagger}(\zeta) d \zeta+\check{h}_{1} \check{h}_{2}^{\dagger}\right] .
$$

The rationale behind this approach is that it is computationally advantageous to use the rescaled eigenfunctions instead of the original ones.

With the inner product defined we can state the normalisation conditions for the eigenfunctions and their adjoints. These are given by

$$
\left\langle v_{l}, v_{l}\right\rangle(\tau) \equiv 1, \quad\left\langle v_{k}^{*}, v_{l}\right\rangle(\tau) \equiv \delta_{k l},
$$

where $\delta_{k l}$ denotes the Kronecker delta.

The expansion given in (3.20) and the approximation in (3.22) constitute an asymptotic solution to the linear problem given in (3.19), and it is expected to be valid provided the eigenvalues remain well separated for all time. Merging or crossing of the eigenvalues requires a separate asymptotic treatment and we do not consider this case here. A discussion about the accuracy of this asymptotic solution, as well as some convergence studies, can be found in Hennessy \& Münch (2013).

\section{Results}

We divide the results into three main subsections. First, the initial stability of the system is explored by setting $\tau=0$ in the rescaled frozen-time eigenvalue problem (3.14) and computing curves in parameter 
space for which the real part of the largest eigenvalue is equal to zero. In time-independent stability problems these curves are referred to as neutral stability curves, and we adopt the same terminology in this paper. Moreover, we refer to the eigenvalue with the largest real part as the top eigenvalue.

The neutral stability curves separate parameter space into regions where the real part of the top eigenvalue is either positive or negative and, in the context of the frozen-time analysis, they can be used to identify regions of parameter space where the base state is instantaneously stable or unstable. In the case when the base state is unstable, these curves can also be used to infer which instabilities are occurring at that set of parameter values.

Secondly, once the initial stability of the problem is understood, we then attempt to infer how the stability evolves over long times by examining how the neutral stability curves change with $\tau$. Finally, we explore the long-term evolution of disturbances directly using the asymptotic solution presented in Section 3.2.2.

\subsection{Initial stability of the system}

We begin by setting $\tau=0$ and computing the neutral stability curves which we write as $M=M(a ; C, G)$. In this form they describe, for a given $C$ and $G$, the Marangoni number $M$ that must be exceeded for a disturbance with wavenumber $a$ to grow. Alternatively, for a given value of $M$, these curves enable one to determine the wavenumbers of any growing disturbances. By combining these two views, it is possible to determine the value of $M$ that must be exceeded for any disturbance to grow at time $\tau=0$, and hence it possible to ascertain the initial stability of the system.

The stationary neutral stability curves corresponding to a zero top eigenvalue can be computed analytically and are given by

$$
M=\frac{12 a^{3}(\sinh 2 a-2 a)\left(a^{2} C^{-1}+G\right)}{\left[3 a \cosh a \sinh a-\left(2 a^{2}+3\right) a^{2}-3 \sinh ^{2} a+3 a^{3} \operatorname{coth} a\right]\left(a^{2} C^{-1}+G\right)+24 a^{5}(a-\operatorname{coth} a)} .
$$

In the limit $C \rightarrow 0$ or $G \rightarrow \infty$, corresponding to strong surface tension or strong gravity, respectively, (4.1) reduces to

$$
M_{\infty}=\frac{12 a^{3}(\sinh 2 a-2 a)}{3 a \cosh a \sinh a-\left(2 a^{2}+3\right) a^{2}-3 \sinh ^{2} a+3 a^{3} \operatorname{coth} a},
$$

which recovers the results from Hennessy \& Münch (2013) for non-deformable fluid layers. This limit will be discussed in more detail below. For finite $G$ and $C>0$ we also have $M \simeq M_{\infty}$ for $a \gg 1$. Thus, for large wavenumbers the dynamics of this system are expected to be similar to those when the free surface is non-deformable.

The limiting neutral stability curve in (4.2) is shown as the solid line in Figure 3, and this curve separates the $(a, M)$ plane into two distinct regions. For Marangoni numbers below the critical value of 80 , the top eigenvalue of the frozen-time linear stability problem (3.14) is always negative and hence the base state is always stable. However, for $M>80$, there is a band of wavenumbers $0<a<a_{1}(M)$ for which the top eigenvalue has a positive real part and the base state is unstable. The wavenumbers $a_{1}$ are those that lie on the neutral stability curve. Moreover, the top eigenvalue is always real when the free surface is held flat and there are no oscillatory instabilities (Hennessy \& Münch, 2013).

The behaviour of the stationary neutral stability curve for $O(1)$ wavenumbers changes drastically when both $G<\infty$ and $C>0$, which is shown by the dashed line in Figure 3 for the example parameter 


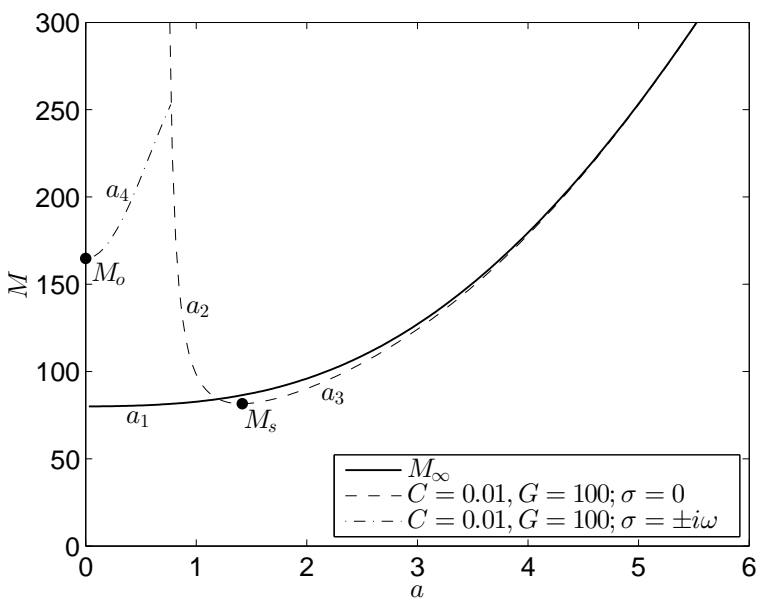

FIG. 3. Neutral stability curves for non-deformable systems (solid black) and deformable systems (dashed and dashed-dot) The solid and dashed curves correspond to locations in parameter space where the top eigenvalue is zero. The dashed-dot line corresponds to where the top eigenvalue is purely imaginary. See text for definitions of $M_{s}, M_{o}$, and the $a_{i}$.
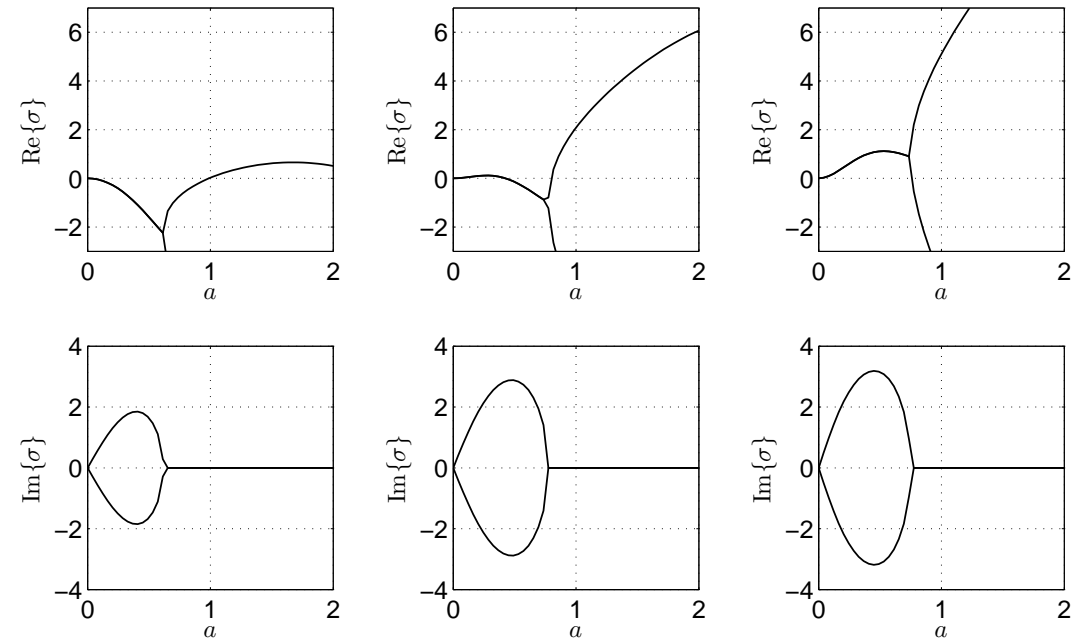

(a) $M=100 \in R_{u, 1}$

(b) $M=200 \in R_{u, 2}$

(c) $M=300 \in R_{u, 3}$

FIG. 4. The dependence of the top two eigenvalues on the disturbance wavenumber for various effective Marangoni numbers. The values of $C$ and $G$ are chosen to coincide with the neutral stability diagram in Figure 3, thus $C=0.01$ and $G=100$. Each value of $M$ illustrates a qualitatively distinct behaviour of the eigenvalues and each behaviour characterises a subregion of the neutral stability diagram. See text for full details. The definitions of the $R_{u, i}$ are given in (4.5). 
values $G=100$ and $C=0.01$. In this case the denominator of (4.1) has a zero while the numerator remains positive, resulting in a singularity in the neutral stability curve when $a=a_{s} \simeq 0.7$. This singularity divides the neutral stability curve into two sections, and the behaviour of the neutral stability curves near this point can be characterised by the following limits:

$$
\lim _{a \rightarrow a_{s}^{-}} M(a ; G, C)=-\infty, \quad \lim _{a \rightarrow a_{s}^{+}} M(a ; G, C)=+\infty .
$$

Thus, the neutral stability curve to the right of the singularity exists only in the upper-quarter plane and it resembles the shape of a " $\mathrm{u}$ " where the left branch is constrained by the vertical asymptote at $a_{s}$ and the right branch tends towards (4.2) for large $a$. The shape of the curve implies the existence of a band of unstable wavenumbers with positive top eigenvalues given by $a_{s}<a_{2}(M)<a<a_{3}(M)$. Moreover, there is a critical value of $M=M_{s} \simeq 82$ such that $a_{2}\left(M_{s}\right)=a_{3}\left(M_{s}\right)$, and this corresponds to the minimum of the neutral stability curve in the upper-quarter plane.

The other section of the stationary neutral stability curve lies to the left of the singularity and it corresponds to negative values of the Marangoni number. Although this indicates the presence of a stationary instability when $M<0$, we do not investigate this situation here.

To explore the dynamics of the system to the left of the singularity and in particular, for small wavenumbers $a$, we compute a long-wave expansion of the top eigenvalue by writing it as a power series of $a$ and then expanding its characteristic equation for $a \ll 1$. We find that the top eigenvalue has the form

$$
\sigma \sim \pm i a \sigma_{1}+a^{2} \sigma_{2} \pm i a^{3} \sigma_{3}+a^{4} \sigma_{4}+O\left(a^{5}\right)
$$

where

$$
\sigma_{1}=\sqrt{M / 2}, \quad \sigma_{2}=5 M / 48-1 / 2-G / 6 \text {. }
$$

This expansion is valid for $a \lesssim \min \left\{M^{1 / 2}, G^{-1 / 2}, C^{-1 / 4}\right\}$. The functions $\sigma_{3}$ and $\sigma_{4}$ are complicated and no insight is gained by writing them here, although we note that they are real valued when $M>0$. From this expansion it can be seen that for $M>0$ the leading-order part of the top eigenvalue is complex and as $M$ is increased beyond $M_{o}=(24+8 G) / 5$, the leading-order contribution to the real part of the eigenvalue changes sign. This indicates there is another neutral stability curve corresponding to the locations in parameter space where only the real part of the top eigenvalue is zero. The smallwavenumber approximation to this curve can be found by solving the expression $\sigma_{2}+a^{2} \sigma_{4}=0$ for $M$, and for larger values of $a$ it can be computed by a numerical continuation scheme.

The oscillatory neutral stability curve is shown as the dashed-dot line in Figure 3. This curve increases monotonically with $a$ from its value of $M_{o}=165$ when $a=0$ until it collides with the left-most branch of the stationary neutral stability curve when $M=253$. Thus, for $M>165$, we expect there to be a band of wavenumbers with complex conjugate eigenvalues with positive real part, thus signifying the presence of an oscillatory instability in the system.

To understand when each mode of instability occurs and for which wavenumbers, we examine the neutral stability curves shown in Figure 3. These curves divide the parameter space into four regions, each of which corresponds to a distinct behaviour of the top eigenvalue as the wavenumber $a$ is varied. 
These regions are characterised by the effective Marangoni number $M$ and are given by

$$
\begin{aligned}
R_{s} & =\left\{M: 0<M<M_{s}\right\}, \\
R_{u, 1} & =\left\{M: M_{s}<M<M_{o}\right\}, \\
R_{u, 2} & =\left\{M: M_{o}<M<253\right\}, \\
R_{u, 3} & =\{M: 253<M\},
\end{aligned}
$$

where $M_{S} \simeq 82$ and $M_{o} \simeq 165$ denote the Marangoni numbers associated with the onset of the stationary and oscillatory instability when $G=100$ and $C=0.01$, respectively. In $R_{S}$ the real part of the top eigenvalue is always negative and hence the base state is stable for these values of the Marangoni number. In the three other regions $R_{u, i}, i=1,2,3$, the real part of the top eigenvalue is positive for some wavenumbers. However, the top eigenvalue exhibits markedly different behaviour within each of these regions. This is demonstrated in Figure 4, where the dependence of the top two eigenvalues on the wavenumber is displayed for three exemplary values of the Marangoni number that characterise each of the three regions. From this figure we see that the top eigenvalues are always a complex conjugate pair for sufficiently small wavenumbers, but as the wavenumber increases the imaginary parts eventually vanish, causing a transition where the complex conjugate pair splits into two real and distinct eigenvalues. The sign of the real parts for small wavenumbers and the sign of the real part when the transition happens distinguishes the three regions.

In the region $R_{u, 1}$ (Figure 4 (a)), the complex eigenvalues always have negative real parts including when they undergo the transition to real eigenvalues. After this transition happens, the largest of the two eigenvalues grows to become positive, giving rise to the stationary instability that occurs for wavenumbers in the range $a_{2}(M)<a<a_{3}(M)$.

The behaviour of the top eigenvalue in $R_{u, 2}$ (Figure 4 (b)) is very similar to in $R_{u, 1}$, except there is now a small band of wavenumbers given by $0<a<a_{4}(M)$ where the real parts of the complex eigenvalues are positive. For $a>a_{4}(M)$ the behaviour of the top eigenvalue is qualitatively identical to in $R_{u, 1}$.

The region $R_{u, 3}$ (Figure 4 (c)) is defined by the fact that the complex eigenvalues always have positive real parts. Thus, when the transition to real eigenvalues occur, the largest grows to become even more positive, whereas the smaller of the two decreases, eventually becoming negative when $a$ is increased beyond $a_{2}(M)$.

From the analysis of the eigenvalues in these regions, we can conclude that only the stationary instability occurs in $R_{u, 1}$, whereas both modes of instability occur in $R_{u, 2}$ and $R_{u, 3}$.

We now revisit the non-deformable limit by studying how the neutral stability curves collapse onto the $M_{\infty}$ curve when surface tension or gravity is strong, i.e., when $C \rightarrow 0$ or $G \rightarrow \infty$, respectively. It can be shown that the location of the singularity in the stationary neutral stability curve is given approximately by $a_{s}^{2} \simeq\left(-G C+\sqrt{G^{2} C^{2}+480 C}\right) / 2$ when $C \ll 1$ or $G \gg 1$ or when both of these are true. Furthermore, the wavenumber that marks the minimum of the stationary neutral stability curve is given by $a_{c} \simeq(4725 / G)^{1 / 4}$ when $G \gg 1$ and $a_{c} \simeq(9450 C)^{1 / 6}$ when $C \ll 1$. Therefore, when $C \rightarrow 0$ or $G \rightarrow \infty$, both the singularity and the minimum of the stationary neutral stability curve tend towards $a=0$. The implication is that the entire left branch of this curve, i.e., the branch defined by $a_{2}$, effectively vanishes in this limit. Moreover, because the oscillatory neutral stability curve exists in the region to the left of the $a_{2}$ branch, it is eliminated in this limit as well. Thus, as $C \searrow 0$ or $G \nearrow \infty$, the region of complex eigenvalues with positive real parts gradually shrinks until it is completely eliminated. 
4.1.1 Competing modes of instability When the parameters are set to $C=0.01$ and $G=100$, the previous analysis has shown the existence of complex eigenvalues with positive real part when the disturbance wavenumber is small. While this does indicate that it may be possible to observe longwavelength oscillatory instabilities in the system, it is important to remember that for a given set of parameter values, the dominant mode of instability, if there is one, is determined by the eigenvalue that has the largest real part over all of the disturbance wavenumbers and the corresponding eigenfunction. Thus, an examination of Figure 4 reveals that, at least initially, an oscillatory instability is unlikely to be observed because the real parts of the complex eigenvalues are always much less than the purely real eigenvalues. To reverse the situation so that the oscillatory instability is dominant, the complex eigenvalues need to have real parts that are larger than the purely real eigenvalues. However, given that the real eigenvalues appear to grow much more rapidly with $M$ than the complex eigenvalues (see Figure 4), it is difficult to satisfy this condition if both instabilities occur simultaneously for a given Marangoni number. This suggests that the oscillatory instability is likely to be dominant only if its onset occurs at lower values of the Marangoni number compared to the stationary mode. We now investigate whether such situations actually arise.

The Marangoni numbers that are associated with the onset of the instabilities are characterised by the minima of the stationary and oscillatory neutral stability curves. The locations of these two minima depend on the other parameter of the system, namely the effective Galileo and capillary numbers $G$ and $C$, respectively. Thus, by tuning these parameters it should be possible to induce the oscillatory instability before the stationary one. We denote by $M_{o}$ and $M_{S}$ the Marangoni numbers that correspond to the minima of the oscillatory and stationary neutral stability curves, respectively. As previously mentioned, the oscillatory neutral stability curve can be approximated for small wavenumbers by solving the equations $\sigma_{2}+a^{2} \sigma_{4}=0$ for $M$, where the $\sigma_{i}$ are defined in (4.4). It can be shown that the oscillatory neutral stability curve has a local minimum at $a=0$, and direct numerical calculations of this curve shows that this point is, in fact, the global minimum. Therefore, we have

$$
M_{o}=\frac{24+8 G}{5}
$$

The minima of the stationary neutral stability curves can, in theory, be found directly from (4.1). However, the wavenumbers that minimise this curve satisfy a cumbersome transcendental equation and hence they must be found numerically.

The parameter dependence of these two minima is shown in Figure 5 (a). In particular, both of these minima are plotted as functions of the Galileo number $G$, and several plots of $M_{S}$ are shown for different values of the capillary number $C$. Thus, for a given value of $C$ and $G$, this figure shows the two critical Marangoni numbers that correspond to the onset of the two types of instability. Parameter regimes where only the oscillatory mode of instability occurs can be identified as those which satisfy $M_{o}(G)<M<M_{S}(C, G)$. An examination of Figure 5 (a) shows that for each value of $C$, the curves $M_{o}=M_{o}(G)$ and $M_{s}=M_{s}(G)$ always intersect at one particular value of $G$, denoted by $G_{p}$. Moreover, for $G<G_{p}(C)$, the condition $M_{o}<M_{s}$ holds. The dependence of $G_{p}$ on $C$ is shown in Figure 5 (b) and the region under this curve corresponds to the parameter regime where the oscillatory instability can potentially be isolated. The non-monotonicity of $G_{p}$ arises because the growth of $M_{s}$ with decreasing $C$ (for fixed $G$ ) is not monotonic either. As can be seen from Figure 5 (a), decreasing $C$ from $10^{-1}$ to $10^{-3}$ increases $M_{s}$ to approximately 84 for all $G$. However, as the non-deformable limit must be recovered as surface tension gets arbitrarily strong, we have that $M_{s} \rightarrow 80$ as $C \rightarrow 0$, i.e., $M_{s}$ eventually begins to decrease. Setting $M_{o}=M_{s}=80$ and solving for $G_{p}$ yields $G_{p} \simeq 47$, which is the asymptote that is approached in Figure 5 (b) as $C$ is decreased to zero. For capillary numbers in the range $C<10^{-2}$, 


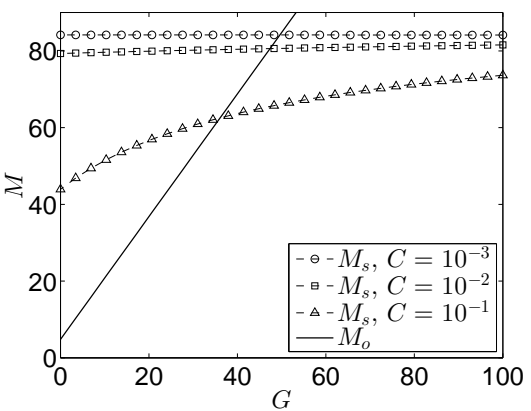

(a)

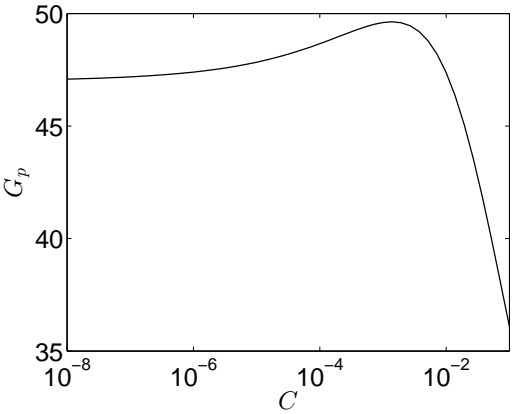

(b)

FIG. 5. Left (a): Critical Marangoni numbers $M$ as a functions of the effective Galileo number $G$ and the effective capillary number $C$. The solid black line denotes the Marangoni numbers associated with the onset of an oscillatory instability and is given analytically by (4.6). The dashed lines with symbols denote Marangoni numbers associated with the onset of stationary instabilities. For each value of $C$ these curves intersect at one particular value of $G$ denoted by $G_{p}$. Right (b): The dependence of the function $G_{p}$ on the capillary number $C$. For $G<G_{p}$, the inequality $M_{o}<M_{s}$ is satisfied. Thus, the region below this curve corresponds to parameter regimes where it is possible to only induce the oscillatory mode of instability.

we have that $G_{p}$ is always greater than 47 and the onset of the stationary instability occurs for $M>80$. Thus, the following rule of thumb can be established: the stationary instability can be suppressed and the oscillatory instability can be induced if $C, G$ and $M$ are chosen such that

$$
C<10^{-2}, \quad G<47, \quad M_{o}<M<80 .
$$

For small but moderate values of $C$, i.e., for $10^{-2}<C \ll 1$, the value of $G_{p}$ will be less than 47 and the upper bound on $M$ will have to be replaced by its corresponding value of $M_{s}$. If $G>G_{p}$, or if $M>M_{s}$, the stationary instability is expected to dominate, if it occurs at all.

\subsection{Evolution of the neutral stability curves}

Having identified the regions of parameter space where the oscillatory instability is initially dominant, we now seek to understand how these regions evolve with changes in the slow time variable $\tau$. Of particular interest is determining the persistence of the region where the oscillatory instability dominates, as this will be important for assessing how likely it is for this instability to be observed experimentally.

The evolution of the stability regions can be monitored through the $\tau$ dependence of the neutral stability curves. This dependence can be introduced in a physically intuitive manner by replacing the effective non-dimensional numbers $M, C$, and $G$ with their original, time-independent, counterparts given in (3.15). In this case the neutral stability curves can be written in terms of the original parameters as

$$
\mathrm{Ma}=F^{-1} \bar{h}^{-3} M\left(\bar{h} k ; \bar{h}^{-1} \mathrm{Ca}, \bar{h}^{3} \mathrm{Ga}\right),
$$

where the $\tau$ dependence of $\bar{h}$ and $F$ has been omitted for brevity. For stationary neutral stability curves the $M$ in the above equation is given by (4.1), whereas the oscillatory neutral stability curves are computed numerically. In the discussion that follows it is convenient to parameterise the slow evolution of 
the neutral stability curves according to $\bar{h}$ instead of $\tau$. The two parameterisations are equivalent owing to the monotonicity of the function $\bar{h}(\tau)$.

An example of how the neutral stability curves evolve with the base state film thickness $\bar{h}$ is shown in Figure 6. The parameter values are $\mathrm{Ca}=0.01, \mathrm{Ga}=30$, and $\beta=0.85$, and they are chosen such that there is a band of Marangoni numbers $53<\mathrm{Ma}<80$ for which only the oscillatory instability occurs when $\tau=0$. The figure shows that as $\bar{h}$ decreases (corresponding to increasing $\tau$ ), the stationary neutral stability curve moves upward which suggests that for these values of $\mathrm{Ca}$ and $\mathrm{Ga}$, the instability is weakened as time progresses. This can be seen by fixing a value of Ma that lies above the minimum of the initial stationary neutral stability curve. As $\tau$ increases the stationary curve moves upwards and eventually its minimum will grow beyond the fixed value of Ma, therefore implying that the stationary instability no longer occurs for this value of the Marangoni number. Interestingly, the situation for the oscillatory neutral stability curve is the opposite, at least initially. That is, Figure 6 shows that as $\bar{h}$ decreases, the oscillatory curve moves downwards, thus suggesting an amplification of this instability. This amplification can be verified by transforming the long-wave eigenvalues given in (4.4) into the original frozen-time eigenvalues via the rescaling in (3.13) and (3.15), which shows that the real part increases as $\tau$ is increased from zero. The downwards motion of the oscillatory curve also means that some Marangoni numbers that are initially in the stable regime, i.e., those below the value of 53 , can enter the unstable regime. The decrease in the oscillatory curve is only temporary, however, and eventually it begins to increase. As $\bar{h}$ tends towards its steady state value of $1-\beta$, both neutral stability curves tend to infinity.

The behaviour of the neutral stability curves can be captured by plotting how their minima evolve with $\bar{h}$, and Figure 7 (a) shows this for the case described above when $\mathrm{Ca}=0.01, \mathrm{Ga}=30$, and $\beta=0.85$. In the figure $\mathrm{Ma}_{\mathrm{s}}$ and $\mathrm{Ma}_{\mathrm{o}}$ denote the critical Marangoni numbers associated with the stationary and oscillatory neutral stability curve, respectively. The curve for $\mathrm{Ma}_{\mathrm{o}}$ can be obtained analytically by rescaling (4.6) to yield

$$
\mathrm{Ma}_{\mathrm{o}}=\frac{\beta}{\bar{h}-1+\beta}\left(\frac{24+8 \bar{h}^{3} \mathrm{Ga}}{5}\right),
$$

and $\mathrm{Ma}_{\mathrm{s}}$ is found numerically. From the figure it is easy to see that $\mathrm{Ma}_{\mathrm{s}}$ increases with time whereas $\mathrm{Ma}_{\mathrm{o}}$ decreases until $\bar{h} \simeq 0.46$, after which it starts to increase rapidly. This behaviour has important physical consequences as it implies that the initial band of Marangoni numbers for which only the oscillatory instability occurs is preserved throughout much of the evolution. Thus, if an experiment is performed that has a Marangoni number within this band, the oscillatory instability will not become dominated by the stationary instability, nor is it expected to quickly stabilise. In contrast, the monotonic increase of $\mathrm{Ma}_{\mathrm{s}}$ with time indicates that the stationary instability will quickly stabilise unless the Marangoni number is much larger than $\mathrm{Ma}_{\mathrm{s}}$.

An analysis of the analytical expression for $\mathrm{Ma}_{\mathrm{o}}$ given in (4.9) reveals that the oscillatory neutral stability curves do not always have to decrease initially. In fact, there is a relationship between $\beta$ and $\mathrm{Ga}$ that determines the initial behaviour of this curve. If we think of $\mathrm{Ga}$ as being fixed for a given set of experiments, then it is possible to define a critical value of the initial solvent volume fraction $\beta$ that separates the two behaviours. In particular, we let

$$
\beta_{c}=\frac{1}{3} \frac{3+\mathrm{Ga}}{\mathrm{Ga}},
$$




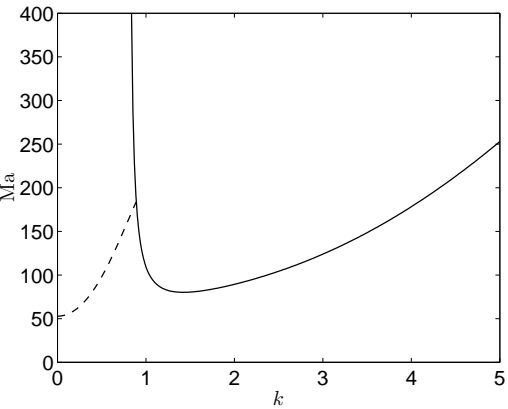

(a) $\bar{h}=1.0$

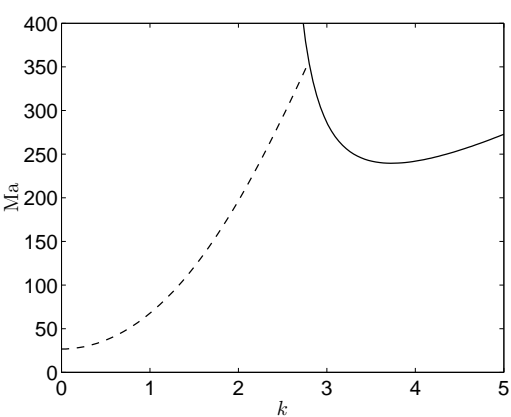

(c) $\bar{h}=0.4$

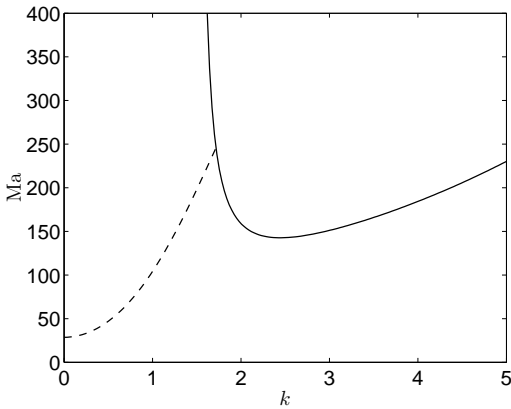

(b) $\bar{h}=0.6$

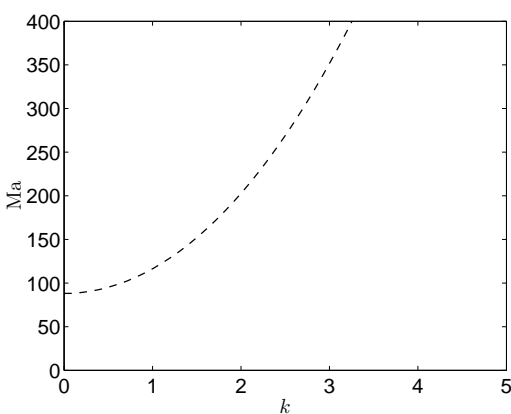

(d) $\bar{h}=0.2$

FIG. 6. Evolution of the neutral stability curves in the upper-quarter plane in terms of the base state film thickness $\bar{h}$. Stationary neutral stability curves are shown as solid black lines and oscillatory ones as dashed lines. Parameter values are $\mathrm{Ca}=0.01$, $\mathrm{Ga}=30$, and $\beta=0.85$. As $\bar{h} \rightarrow 1-\beta=0.15$ the curves tend to infinity.

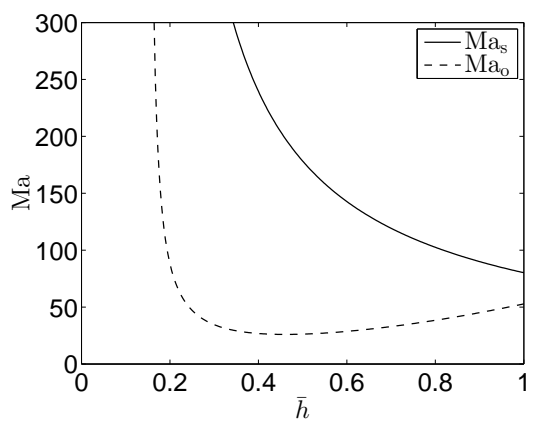

(a)

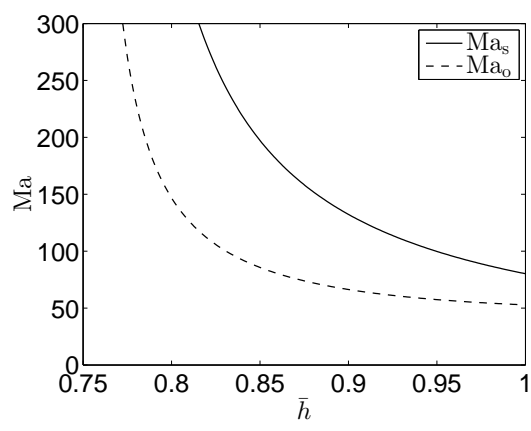

(b)

FIG. 7. The evolution of the minima of the stationary (solid) and oscillatory (dashed) neutral stability curves. The parameter values are (a): $\mathrm{Ca}=0.01, \mathrm{Ga}=30, \beta=0.85 ;(\mathrm{b}): \mathrm{Ca}=0.01, \mathrm{Ga}=30, \beta=0.25$. Note the difference in scales in panel (b). 
and if $\mathrm{Ga}>3 / 2$, then values of $\beta$ such that $\beta_{c}<\beta<1$ will yield an initially decreasing oscillatory neutral stability curve. In the case when $\mathrm{Ga}<3 / 2$ the curve always increases. When $\mathrm{Ga} \gg 1$, as in the case of the experiments by Bassou \& Rharbi (2009), we have $\beta_{c} \simeq 1 / 3$. Figure 7 (b) shows an example of $\mathrm{Ma}_{\mathrm{o}}$ increasing with time for the example parameter values of $\mathrm{Ca}=0.01, \mathrm{Ga}=30$, and $\beta=0.25$. The value of $\beta_{c}$ in this case is $\beta_{c} \simeq 0.37$.

The results from this analysis indicate that if $\mathrm{Ga}$ is chosen so that there is an initial band of Marangoni numbers for which only the oscillatory instability occurs and if $\beta$ is above the critical value defined in (4.10), then this band of Marangoni numbers will indeed persist for some time, provided that $\mathrm{Ma}_{\mathrm{s}}$ increases with $\tau$. While it is not easy to formulate analytical conditions which guarantee the increase of $\mathrm{Ma}_{\mathrm{s}}$, numerical experiments in the regime $\mathrm{Ca} \ll 1$ have suggested this is always the case.

\subsection{Disturbance evolution}

The analysis based on the frozen-time method has shown the presence of an oscillatory instability and it enabled us to identify regions in parameter space where this instability temporarily dominates. We now use refined methods to study the evolution of disturbances in more detail. More specifically, we examine the amplification of disturbances over long periods of time using the asymptotic solution in Section 3.2.2 and by numerically solving (3.19) as an initial value problem. The numerical scheme is similar to the one used for approximating solutions to the base state; space is discretised using second-order finite differences and the resulting problem is treated as a system of ODEs. We use the long-time asymptotic solution for the base state (3.6) in the numerical solution of the linearised problem to be consistent with our previous analysis. The effects of including transients in the base state are discussed in Hennessy \& Münch (2013). Using the asymptotic and numerical solutions, the amplification of a disturbance at time $t$ is defined as

$$
A(t)=\frac{\left.\max \{\| \tilde{c}(z, t)\}_{\infty},|\tilde{h}(t)|\right\}}{\max \left\{\left\|\tilde{c}_{i}(z)\right\|_{\infty},\left|\tilde{h}_{i}\right|\right\}},
$$

where $\|\cdot\|_{\infty}$ denotes the usual max norm over the spatial domain. Generally, the initial condition for the disturbance is chosen to be the top eigenfunction at time $\tau=0$. This choice is expected to produce near-maximal amplifications by minimising the contributions from subdominant modes. The asymptotic solution in Section 3.2.2 is truncated and only the first two modes are considered. This allows us to deal with the case when the top eigenvalues are a complex conjugate pair and both corresponding modes need to be included in the asymptotic solution.

We first investigate the disturbance amplifications that result from the oscillatory instability. We set $\mathrm{Ma}=70$, and take the other parameters to be

$$
\mathrm{Ca}=0.01, \quad \mathrm{Ga}=30, \quad \beta=0.85, \quad \delta=0.01 .
$$

These parameters correspond to the situation shown in Figure 6, where disturbances with wavenumbers that satisfy $k<0.31$ are expected to grow in an oscillatory fashion until the system begins to stabilise.

Figure 8 shows the evolution of the top two eigenvalues, the real part of disturbance to the film thickness measured relative to its initial value, and the disturbance amplification when the wavenumber is set to $k=0.21$. This particular wavenumber is chosen because it corresponds to the eigenvalue with the largest real part when $\tau=0$. For these parameters values the disturbance is amplified by more than three orders of magnitude before the system begins to stabilise, which may be large enough for this instability to be observed experimentally. 
An examination of the top eigenvalues in Figure 8 shows that the complex conjugate pair eventually splits into two distinct real eigenvalues because the imaginary parts tend toward zero after an initial period of growth. This influences the behaviour of the oscillations and it explains the behaviour of the disturbance to the film thickness in the middle panel of Figure 8. More specifically, the increasing magnitude of the imaginary part of the eigenvalue leads to an increase in the oscillation frequency, but its eventual decay to zero causes the frequency to decrease until the oscillations are completely suppressed.

As the imaginary parts of the top eigenvalues tend toward zero, the distance between the two eigenvalues decreases as well. At the point where the imaginary part vanishes, the two eigenvalues are identical and thus, the asymptotic solution in Section 3.2.2 breaks down. This can be clearly seen in Figure 8; in particular, as the critical value of $\tau$ is approached the asymptotic solution diverges. Before this breakdown occurs, however, the asymptotic solution matches the numerical simulation with a very high degree of accuracy. The stabilisation of the system and the long-term dynamics are accurately captured, and the error in the maximum amplification is less than $0.1 \%$.

For larger values of Ma that still satisfy $\mathrm{Ma}<\mathrm{Ma}_{\mathrm{s}}$, the maximum disturbance amplification caused by the oscillatory instability can be further increased while the onset of the stationary instability is still inhibited. Thus, by increasing Ma up to this critical value it is possible to strengthen the oscillatory instability while maintaining its dominance. For $\mathrm{Ma}>\mathrm{Ma}_{\mathrm{s}}$ the stationary instability also occurs; however, the frozen-time analysis suggested this instability quickly stabilises which might allow the oscillatory instability to remain dominant. Indeed, when $\mathrm{Ma}=90$, keeping the other parameters as in (4.12), the oscillatory mode with wavenumber $k=0.3$ is amplified by more than four orders of magnitude, whereas the maximum amplification of a stationary mode with wavenumber $k=1.52$ is less than eight times its initial value. The wavenumber $k=0.3$ maximises the real part of the complex eigenvalues when $\tau=0$, and $k=1.52$ maximises the purely real eigenvalues. As the Marangoni number is increased further, the oscillatory modes still obtain larger overall amplifications, but the rapid initial growth of the stationary modes means they obtain larger amplifications in a shorter amount of time. For example, when $\mathrm{Ma}=100$ a stationary mode with wavenumber $k=1.68$ obtains a maximum amplification of roughly 5000 , whereas an oscillatory mode with wavenumber $k=0.34$ has only been amplified by a factor of about 550 in the same amount time. Figure 9 shows this situation in more detail. Again, these wavenumbers are chosen to maximise the real parts of the eigenvalues when $\tau=0$, thus ensuring these modes have the fastest initial growth. For $\mathrm{Ma} \gg \mathrm{Ma}_{\mathrm{s}}$ we can conclude that the switch in dominance occurs not because the stationary modes obtain larger overall amplifications before the stabilisation occurs, but because they are amplified much more rapidly than the oscillatory modes.

The frozen-time analysis predicted that when $\mathrm{Ga}=30, \mathrm{Ca}=0.01$, and $\beta=0.85$, it was possible for the oscillatory instability to occur for Marangoni numbers that are less than the initial critical value of $\mathrm{Ma}_{\mathrm{o}} \simeq 53$. This raises the question of whether there is a smaller critical Marangoni that could be used to denote the onset of the instability. In the case when $\mathrm{Ma}=53$ and $\delta=0.01$, we find that disturbances only obtain moderate amplifications with the largest being approximately 150 . Moreover, the maximum amplification rapidly decreases with $\mathrm{Ma}$; for example, when $\mathrm{Ma}=45$ we have $\max A(t) \simeq 12$ for the initial disturbances we consider. Therefore, the instabilities that occur for $\mathrm{Ma}<\mathrm{Ma}_{\mathrm{o}}$ are unlikely to have any significant physical relevance and the critical Marangoni number is not expected to be below its initial value of $\mathrm{Ma}_{\mathrm{o}} \simeq 53$.

We can compare the initial critical Marangoni number of 53 for the example parameters in (4.12) to the critical value that is predicted when the surface is assumed to be non-deformable. Hennessy \& Münch (2013) consider this case and they find that the critical value is at least 80 . This is considerably 

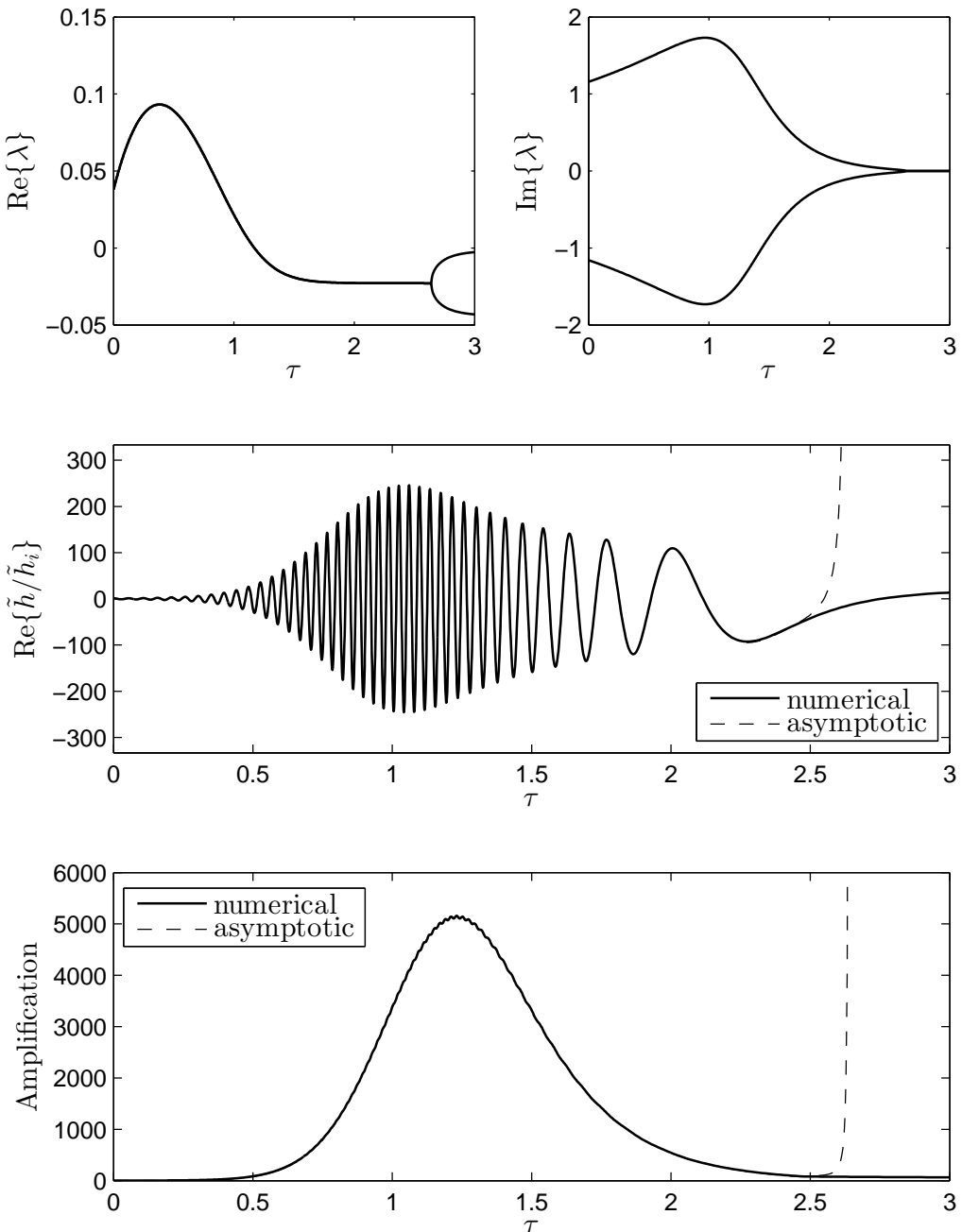

FIG. 8. The oscillatory instability. Top (a): The evolution of the real and imaginary parts of the top two eigenvalues. Middle (b): Evolution of the real part of the disturbance to the film thickness relative to its initial value. Bottom (c): Disturbance amplification as a function of time. The asymptotic solution in Section 3.2.2 breaks down and diverges as the imaginary parts of the eigenvalues tend toward zero. Parameter values are $\mathrm{Ma}=70, \mathrm{Ca}=0.01, \mathrm{Ga}=30, \beta=0.85, \delta=0.01$, and $k=0.21$. The top eigenfunction at $\tau=0$ is used as the initial condition. 


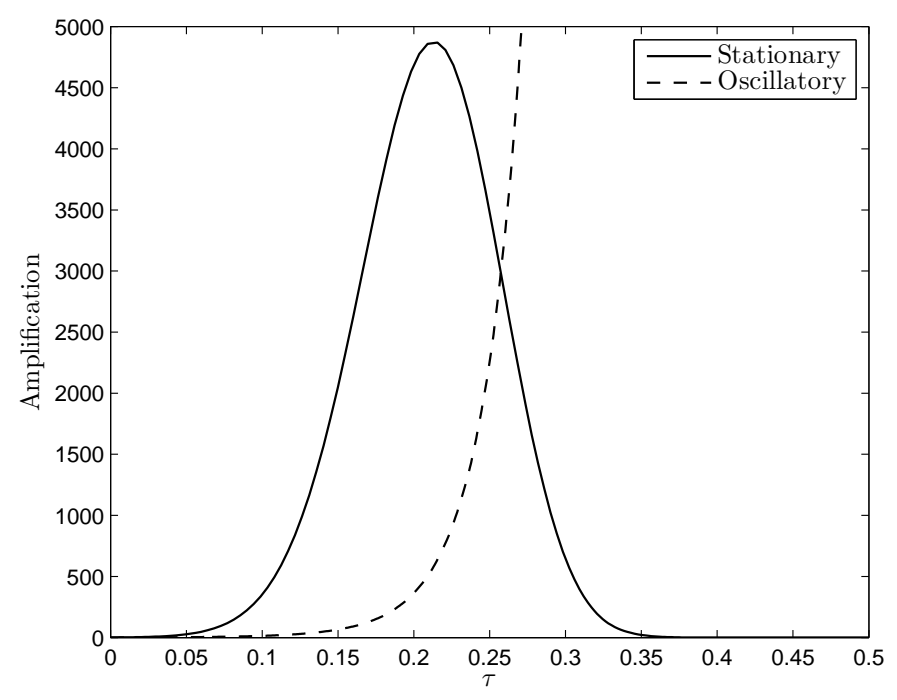

FIG. 9. Numerically computed amplifications of stationary (solid) and oscillatory (dashed) modes when Ma $=100$, Ga $=30$, $\mathrm{Ca}=0.01, \beta=0.85$, and $\delta=0.01$. The stationary mode has wavenumber $k=1.68$ whereas the oscillatory mode has $k=0.34$ and the initial condition is taken to be the top eigenfunction at time $\tau=0$. While the oscillatory mode obtains a larger overall amplification, the rapid growth of the stationary mode means that it can obtain much larger initial amplifications.

larger than our value of 53, and Figure 5 shows that the critical Marangoni number can likely be made even smaller by further decreasing Ga. Thus, films with deformable upper surfaces are much more susceptible to Marangoni instabilities, particularly when gravity is weak and surface tension is strong.

\section{Application to experiments}

The results from the previous section have shown that the two modes of instability will always occur simultaneously for sufficiently large values of the Marangoni number Ma, but generally in this case the stationary mode dominates because it amplifies disturbances much more rapidly than the oscillatory mode. However, for certain parameters the stationary instability can be suppressed in the sense that it occurs at Marangoni numbers that are larger than those needed to induce the oscillatory instability. This is found to occur, for instance, when both the capillary and Galileo numbers are small. The corresponding physical situation in this case would have sufficiently strong surface tension to inhibit the stationary instability and weak gravitational effects to promote the oscillatory one. The effects of gravity can be reduced, for example, by using low-density mixtures or by performing experiments in a microgravity environment.

There are practical limits to how strong the effects of surface tension can be, and this is partly due to the fact that range of wavenumbers that are in the unstable, oscillatory regime tends to decrease with the capillary number. In the extreme case when the capillary number tends towards zero, this range of wavenumbers vanishes and the oscillatory instability is completely suppressed. Thus, if surface tension is too strong the wavelength of the unstable oscillatory modes could be larger than the dimensions of the vessel containing the mixture. Another aspect to consider is that strong surface tension will tend to 
reduce the amplitude of surface deflections, perhaps increasing the difficulty of detecting the presence of the oscillatory instability.

From the definition of the capillary and Galileo numbers (see (2.16) and (2.19)), it is clear that a simple way to vary the effective strength of surface tension and gravity is by changing the initial thickness of the fluid layer. Moreover, from the discussion in Section 4.1.1, the oscillatory instability has the potential to be dominant when $\mathrm{Ca}<10^{-2}$ and $\mathrm{Ga}<47$. Thus, by imposing these conditions we can determine a range of film thicknesses where the oscillatory instability will temporarily dominate provided the Marangoni number is not too large. This range is given explicitly by

$$
\frac{100 \mu D}{\gamma_{r}}<h_{m}<\left(\frac{47 \mu D}{\rho g}\right)^{1 / 3} .
$$

For films that lie outside of this region because they are too thick, gravity causes the onset this instability to occur at larger Marangoni numbers than the stationary instability. If, however, $h_{m}$ is below the lower bound in (5.1), then both modes of instability can dominate. The dominance is determined by whether the Galileo number is above or below the particular value $\mathrm{Ga}_{p}$ defined in Section 4.1.1, and it depends on the size of the Marangoni number.

If $h_{m}$ is chosen in such a way that the inequality in (5.1) is satisfied, then the strength of the Marangoni effects can be controlled through the evaporation rate $k_{m}$. For the oscillatory instability to occur at least initially, the Marangoni number must be greater than $\mathrm{Ma}_{\mathrm{o}}=(24+8 \mathrm{Ga}) / 5$. However, it must be small enough to prevent the onset of the stationary instability; thus, Ma $\lesssim 80$ must also be enforced. These restrictions limit the evaporation rate to lie between the values

$$
\left(\frac{24+8 \mathrm{Ga}}{5}\right) \frac{\mu D^{2}}{\beta(1-\beta) \rho \gamma_{c} h_{m}^{2}}<k_{m}<\frac{80 \mu D^{2}}{\beta(1-\beta) \rho \gamma_{c} h_{m}^{2}} \ll \frac{D}{h_{m}},
$$

where the last inequality arises due to our primary assumption that $\delta \ll 1$.

To further aid in the observation of the oscillatory instability, the initial solvent volume fraction $\beta$ can be chosen to be larger than the critical value of $\beta_{c}$ given in (4.10), which requires that $\mathrm{Ga}>3 / 2$. Under these conditions the critical Marangoni number associated with the onset of the oscillatory instability, $\mathrm{Ma}_{0}$, decreases initially, thus prolonging the duration of the instability.

These results can be discussed in the context of the experiments by Bassou \& Rharbi (2009) by evaluating the inequality in (5.1) using the parameter values listed in Table 1. For the oscillatory instability to occur at lower Marangoni numbers than the stationary instability, the films should have an initial thickness between $690 \mathrm{~nm}$ and $100 \mu \mathrm{m}$. This range lies slightly outside the thinnest films that were studied by Bassou and Rharbi which were $150 \mu \mathrm{m}$ initially. In such films the oscillatory instability would be subdominant which likely explains why only the stationary instability was observed in their experiments.

We can also apply the linear stability results to investigate the dynamics of drying paint films; see, for example, Wilson (1993) and Howison et al. (1997). Although no instabilities are found in these studies, several observations, namely the crest-to-trough reversal that is described in the introduction and the decaying oscillations in numerical simulations, indicate that the parameters associated with paint films might be close to the parameter regime where the oscillatory instability occurs. If we insert the parameter values from Howison et al. (1997) into (5.1), we find that the oscillatory instability should be observed in paint films with an initial thickness between $20 \mu \mathrm{m}$ and $298 \mu \mathrm{m}$, provided the Marangoni number is above the critical value of $\mathrm{Ma}_{0}$. The paint films studied in the aforementioned references fall within this range of thicknesses; for example, $h_{m}=60 \mu \mathrm{m}$ is often used as a characteristic value of the initial 
thickness of the layer. According to our theory, however, these films are linearly stable because of small Marangoni numbers; we find that $\mathrm{Ma} \simeq 1.3 \times 10^{-3}$ whereas the critical value is $\mathrm{Ma}_{\mathrm{o}} \simeq 5.4$. Although the films have the correct initial thickness for the oscillatory instability to occur, they are ultimately stable which could explain the presence of decaying oscillations and a finite number of reversals.

It would be interesting to perform experiments with evaporating layers of paint with greater initial thicknesses and larger evaporation rates. The latter could be made possible by, for example, prescribing an airflow above the layer, although one would have to be certain that the air does not influence the dynamics of the film. Both of these factors will lead to larger Marangoni numbers and it might be possible to surpass the value that is needed to induce the oscillatory instability in the system.

\section{Summary and conclusion}

In this paper the dynamics of an evaporating solvent-polymer mixture with a deformable surface have been studied. The evaporation of solvent can lead to non-uniformities in the concentration of solvent at the surface of the fluid layer and these can induce surface-tension gradients if the surface tension depends on the local composition of the mixture. Sufficiently large gradients in the surface tension can lead to instabilities in the system if they are able to overcome the stabilising effects of viscosity, gravity, and surface tension.

The onset of instability was investigated mathematically within the framework of linear stability theory. Unlike traditional linear stability problems, the base state is time dependent as a result of evaporative mass loss. By assuming that evaporation occurs on a much longer time scale than diffusion, we find that the quasi-stationary evolution of the base state is much slower than the evolution of disturbances to it. We exploit this separation of time scales to study the stability of the base state using frozen-time and asymptotic methods.

The frozen-time method treats the slow time that appears in the base state as a parameter rather than a time variable, thus allowing traditional techniques from linear stability theory to be applied. Using this method is it possible to deduce the instantaneous stability of the system over a wide range of parameter values. The changes in stability that occur over the long periods of time on which the base state evolves are ascertained using an asymptotic solution that is uniformly valid for large times. The asymptotic solution is derived in two different ways using the method of multiple scales and the WKB approximation, and it generalises the solution presented by Hennessy \& Münch (2013). We find that the frozen-time and asymptotic approaches complement each other well, with each method providing crucial insight into the dynamics that occur within the system.

Our analysis indicates that two modes of instability occur in the model. One of these is a stationary mode corresponding to the classical Bénard-Marangoni instability first investigated by Pearson (1958). In this case, surface tension and/or gravity act to keep the surface relatively undeformed. Shear stresses due to surface-tension gradients cause fluid near the surface to flow from regions of low surface tension to high surface tension, i.e., from solvent-rich to solvent-depleted regions. Conservation of mass requires fluid to rise beneath regions of low surface tension and sink near regions of high surface tension, resulting in convection cells. The uprising fluid is rich in solvent but as it is pulled across the surface evaporation depletes it of such. Thus, the regions of low and high surface tension persist and the convective motion is sustained. The second mode of instability is oscillatory and is related to the crest-to-trough reversals that are seen in drying paint layers and waterborne coatings. This mode is due to the combined action of gravity, surface tension, and surface-tension gradients. When a deformed film levels under the action of gravity and surface tension, the concentration of solvent is higher under the crests compared to the troughs. This leads to surface-tension gradients that enhance the levelling 
process. Moreover, these gradients are still present when the film becomes flat, and thus fluid is pulled from regions that used to be crests to regions that used to be troughs. The accumulation and depletion of fluid causes crests to become troughs and vice-versa, and the process then repeats itself.

The two modes of instability are found to compete with each other, with the stationary mode typically being dominant when both occur for the same parameter values. This dominance arises because short-wave cellular modes are amplified more rapidly than long-wave deformational modes. However, in films with strong surface tension, which we characterise by a capillary number condition $\mathrm{Ca}<10^{-2}$, the dominance can be reversed if the Marangoni number is not too large. This is due to the fact that when $\mathrm{Ca}<10^{-2}$, regions of parameter space exist where only the oscillatory mode of instability occurs. A defining feature of these regions is that the onset of the oscillatory mode occurs at lower values of the Marangoni number compared to the stationary mode. The existence and size of these regions depend sensitively on the effective strength of gravity, as measured by the Galileo number Ga. This is because gravity plays a major role in determining the critical Marangoni number associated with the onset of the oscillatory instability. Physically, this strong dependence corresponds to the fact that surface-tension gradients have to pull harder to cause a reversal in heavier fluids; thus, the critical Marangoni number increases with $\mathrm{Ga}$. The exact relation is $\mathrm{Ma}_{\mathrm{o}}=(24+8 \mathrm{Ga}) / 5$. On the other hand, gravity does not play a large role in determining the critical Marangoni number associated with the stationary instability when surface tension is strong. This is due the fact that, in the absence of buoyancy, gravity will only play a role if the fluid surface is deformed. Strong surface tension, however, prevents the short-wave cellular patterns from deforming the surface. For $\mathrm{Ca}<10^{-2}$, our analysis indicates that $\mathrm{Ma}_{\mathrm{s}} \geqslant 80$. Therefore, the region where the oscillatory instability is isolated and, as a result, is dominant can be identified as $\mathrm{Ca}<10^{-2}, \mathrm{Ga}<47$, and $(24+8 \mathrm{Ga}) / 5<\mathrm{Ma}<80$. Using these parameter bounds we have determined experimental conditions such as film thicknesses and evaporation rates that can be used to observe the oscillatory instability.

The onset of both modes of instability and their persistence over long times is likely to be strongly influenced by the rheology of the fluid. In this paper we have assumed the mixture remains Newtonian with a constant viscosity. However, this is generally not true for polymer solutions because they exhibit shear-thinning behaviour after a critical shear rate is exceeded. Below this critical value the Newtonian regime is recovered; see Myers (2005); Chhabra \& Richardson (2008). As shown by Kulicke \& Kniewske (1984) for polystyrene (PS) and toluene mixtures, the critical value of the shear rate can decrease by several orders of magnitude as the molecular weight and concentration of polymer increases. Using their data, we can roughly estimate the relevance of shear-thinning behaviour in this work which is based on the PS-toluene solutions used by Bassou \& Rharbi (2009). The critical shear rate $\dot{\gamma}_{\text {crit }}$ in a $3 \%$ mixture by weight using PS with a (relative) molecular weight of $3.3 \times 10^{5}$ is approximately $10^{4}$ $\mathrm{s}^{-1}$. The PS used by Bassou and Rharbi had a slightly smaller molecular weight of $1.5 \times 10^{5}$, but the initial concentration of polymer was often $15 \%$ by volume (17.6\% by weight). If we assume we can still compare these two mixtures, then a typical shear stress can be estimated using (2.18h) to obtain

$$
\dot{\gamma} \simeq \frac{D}{h_{m}^{2}} \mathrm{Ma}=\frac{k_{m} \beta(1-\beta) \rho \gamma_{c}}{\mu D} .
$$

The order of magnitude of these parameters can be determined from Table 1. Using these to calculate the shear rate, we find that $\dot{\gamma} \simeq 1 \mathrm{~s}^{-1} \ll \dot{\gamma}_{\text {crit }}$. This comparison gives some indication that shear-thinning effects are not important during the early stages of drying when the instability is developing and it provides some justification for the Newtonian rheology used in the mathematical model.

In the absence of non-Newtonian effects, the viscosity of a binary mixture will still depend on the solute concentration. Previous theoretical works have modelled the viscosity as an exponentially 
increasing function of the polymer concentration; see Münch et al. (2011) or Eres et al. (1999). Including this dependence in the current model is expected stabilise the system because the Marangoni effects are effectively weakened as the mixture viscosity increases due to the depletion of solvent. Indeed, the main stabilising mechanism in the paint drying simulations of Eres et al. (1999) seems to be due to a composition-dependent viscosity. Thus, a more comprehensive linear stability analysis that includes realistic fluid rheologies would also aid in determining whether the oscillatory instability found here is physically relevant. The asymptotic solution that was derived in Appendix A would be a helpful tool to carry out such an analysis because it can capture the slow rheological changes that occur during drying process. This serves to further highlight the usefulness and wide range of applicability of such a solution.

\section{Acknowledgements}

The authors would like to thank Chris Breward and Colin Please for insightful discussions about models of evaporating solvent-polymer blends, and Peter Howell and Jon Chapman for suggesting the WKB approach to the linear stability problem.

\section{Funding}

This work was supported by the King Abdullah University of Science and Technology (KAUST) [KUKC1-013-04].

\section{REFERENCES}

BASSOU N. \& RHARBi Y. (2009) Role of Bénard-Marangoni instabilities during solvent evaporation in polymer surface corrugations, Langmuir, 25 (1), 624-632.

BÉnARd H. (1900) Les tourbillons cellulaires dans une nappe liquide, Rev. Gen. Sci. Pures Appl., 11 (1309), 1261-1271.

BÉNARD H. (1901a) Les tourbillons cellulaires dans une nappe liquide transportant de la chaleur par convection en regime permanent, Annales de Chimie et de Physique, 23, 62-144.

BÉNARD H. (1901b) Les tourbillons cellulaires dans une nappe liquide: Méthodes optiques d'observation et d'enregistrement, J. Phys. Theor. Appl., 10 (1), 254-266.

Bender C. \& Orszag S. (1999) Advanced Mathematical Methods for Scientists and Engineers. New York: Springer.

Berg J.C., Boudart M. \& ACrivos A. (1966) Natural convection in pools of evaporating liquids, J. Fluid Mech., 24 (4), 721-735.

BLOCK M.J. (1956) Surface tension as the cause of Bénard cells and surface deformation in a liquid film, Nature, 178, 650-651.

Bodenschatz E., Pesch W. \& Ahlers G. (2000) Recent developments in Rayleigh-Bénard convection, Ann. Rev. Fluid Mech., 32 (1), 709-778.

Bornside D.E., Macosko C.W. \& SCRIVEn L.E. (1989) Spin coating: One-dimensional model, J. Appl. Phys., $66(11), 5185-5193$.

Burelbach J.P., BANKoff S.G. \& DAVIS S.H. (1988) Nonlinear stability of evaporating/condensing liquid films, J. Fluid Mech., 195 (1), 463-494.

Chauvet F., Dehaeck S. \& Colinet P. (2012) Threshold of Bénard-Marangoni instability in drying liquid films, EPL (Europhysics Letters), 99 (3), 34001.

ChHabra R.P. \& Richardon J.F. (2008) Non-Newtonian Flow and Applied Rheology: Engineering Applications, 2nd edn. Oxford: Elsevier. 
Colinet P., Legros J.C. \& Velarde M.G. (2001) Nonlinear Dynamics of Surface-Tension-Driven Instabilities. Berlin: Wiley-VCH.

Craster R.V. \& Matar O.K. (2009) Dynamics and stability of thin liquid films, Rev. Mod. Phys., 81 (3), 11311198.

DAvis S.H. (1976) The stability of time-periodic flows, Ann. Rev. Fluid Mech., 8 (1), 57-74.

DE Gans B.-J., Duineveld P.C. \& SChUbert U.S. (2004) Inkjet printing of polymers: State of the art and future developments, Advanced Materials, 16 (3), 203-213.

Doumenc F., Boeck T., Guerrier B. \& Rossi M. (2010) Transient Rayleigh-Bénard-Marangoni convection due to evaporation: a linear non-normal stability analysis, J. Fluid Mech., 648, 521-539.

Dziwnik M., Korzec M., MÜNCh A. \& WAgner B. (2014) Stability analysis of non-constant base states in thin film equations, SIAM J. Appl. Math., in press.

ERES M.H., WEIDNER D.E. \& SCHWARTZ L. (1999) Three-dimensional direct numerical simulation of surfacetension-gradient effects on the leveling of an evaporating multicomponent fluid, Langmuir, 15 (5), 1859-1871.

GÜNES S., NeUgebauer H. \& SARICIFTCI N.S. (2007) Conjugated polymer-based organic solar cells, Chemical Reviews, 107 (4), 1324-1338.

HAUt B. \& COLInET P. (2005) Surface-tension-driven instabilities of a pure liquid layer evaporating into an inert gas, J. Colloid Interface Sci., 285 (1), 296-305.

HENNESSY M.G. \& MÜNCH A. (2013) A multiple-scale analysis of evaporation induced Marangoni convection, SIAM J. Appl. Math., 73 (2), 974-1001.

HERIOT S.Y. \& JONES R.A.L. (2005) An interfacial instability in a transient wetting layer leads to lateral phase separation in thin spin-cast polymer blend films, Nature Materials, 4 (10), 782-786.

Howison S.D., Moriarty J.A., OCKendon J.R., Terrill E.L. \& Wilson S.K. (1997) A mathematical model for drying paint layers, J. Engrg. Math., 32 (4), 377-394.

KANG K.H. \& CHOI C.K. (1997) A theoretical analysis of the onset of surface-tension-driven convection in a horizontal liquid layer cooled suddenly from above, Phys. Fluids, 9 (7), 7-15.

Kevor Kian J. \& Cole J.D. (1996) Multiple Scale and Singular Perturbation Methods. New York: Springer.

Kojima S., Moriga T. \& TAKenouchi K. (1995) The leveling of thermosetting waterborne coatings. Part III: Leveling under controlled conditions, Polym. Eng. Sci, 35 (24), 1949-1954.

Koschmieder E.L. (1993) Bénard Cells and Taylor Vortices. Cambridge: Cambridge University Press.

KULICKE W.-M. \& KNIEWSKE R. (1984) The shear viscosity dependence on concentration, molecular weight, and shear rate of polystyrene solutions, Rheol. Acta, 23 (1), 75-83.

LICK W. (1965) The instability of a fluid layer with time-dependent heating, J. Fluid Mech., 21 (3), 565-576.

Machrafi H., Rednikov A., Colinet P. \& Dauby P.C. (2010) Bénard instabilities in a binary-liquid layer evaporating into an inert gas, J. Colloid Interface Sci., 349 (1), 331-353.

Machrafi H., Rednikov A., Colinet P. \& Dauby P.C. (2011) Bénard instabilities in a binary-liquid layer evaporating into an inter gas: Stability of quasi-stationary and time-dependent reference profiles, Eur. Phys. $J$. Special Topics, 192 (1), 71-81.

Moons E. (2002) Conjugated polymer blends: linking film morphology to performance of light emitting diodes, J. Phys.: Condens. Matter, 14 (47), 12235-12260.

MÜNCh A., PleAsE C.P. \& WAGNer B. (2011) Spin coating of an evaporating polymer solution, Phys. Fluids, 23 (10), 102101.

Myers T.G. (1998) Thin films with high surface tension, SIAM Review, 40 (3), 441-462.

Myers T.G. (2005) Application of non-Newtonian models to thin film flow, Phys. Rev. E, 72 (6), 066302.

NANAGARA B., O'CONNOR R.D. \& BLUM F.D. (1992) Mobility of toluene in polystyrene-toluene solutions: a NMR study, J. Phys. Chem., 96 (15), 6417-6423.

Oron A., Davis S.H. \& Bankoff S.G. (1997) Long-scale evolution of thin liquid films, Rev. Mod. Phys., 69 (3), 931-980. 
Overdiep W.S. (1986) The levelling of paints, Prog. Org. Coat., 14 (2), 159-175.

PEARSON J.R.A. (1958) On convective cells induced by surface tension, J. Fluid Mech., 4 (5), $489-500$.

PÉREZ-GARCÍA C. \& CARNEIRO G. (1991) Linear stability analysis of Bénard-Marangoni convection in fluids with deformable free surface, Phys. Fluids A, 3 (2), 292-298.

RAYLEIGH L. (1916) On convection currents in a horizontal layer of fluid, when the higher temperature is on the under side, Philos. Mag., 32 (192), 529-546.

SCRIVEN L.E. \& STERnLING C.V. (1964) On cellular convection driven by surface-tension gradients: effects of mean surface tension and surface viscosity, J. Fluid Mech., 19 (3), 321-340.

Serpetsi S.K. \& Yiantsios S.G. (2012) Stability characteristics of solutocapillary Marangoni motion in evaporating thin films, Phys. Fluids, 24 (12), 122104.

Shklyaev S., Khenner M. \& AlabuzheV A.A. (2010) Oscillatory and monotonic modes of long-wave Marangoni convection in thin film, Phys. Rev. E, 82 (2), 025302.

Shklyaev S., AlabuzheV A.A. \& KHENNER M. (2012) Long-wave Marangoni convection in a thin film heated from below, Phys. Rev. E, 85 (1), 016328.

SMith K.A. (1966) On convective instability induced by surface-tension gradients, J. Fluid Mech., 24 (2), $401-$ 414.

SMOLKA L.B. \& WiTELSKI T.P. (2009) On the planar extensional motion of an inertially driven liquid sheet, Phys. Fluids, 21 (4), 042101.

Souche M. \& Clarke N. (2009) Interfacial instability in bilayer films due to solvent evaporation, Eur. Phys. J. $E, 28$ (1), 47-55.

Sultan E., Boudaoud A. \& Amar M.B. (2005) Evaporation of a thin film: diffusion of the vapour and Marangoni instabilities, J. Fluid Mech., 543, 183-202.

TAKASHIMA M. (1981) Surface tension driven instability in a horizontal liquid layer with a deformable free surface. II. Overstability, J. Phys. Soc. Japan, 50 (8), 2751-2756.

VIDAL A. \& ACRIVOS A. (1966) Nature of the neutral state in surface-tension driven convection, Phys. Fluids, 9 (3), 615 .

VRENTAS J.S. \& VRENTAS C.M. (2004) Exchange of stabilities for surface tension driven convection, Chem. Eng. Sci., 59 (21), 4433-4436.

WiLson S.K. (1993) The levelling of paint films, IMA J. Appl. Math. 50 (2), 149-166.

\section{A. Derivation of the asymptotic solution}

We seek to construct a uniformly-valid asymptotic solution to the non-autonomous linear problem given by

$$
\partial_{t} U=L(\tau) U
$$

where $\tau=\delta t$ is a slow time with $\delta \ll 1$, and $L$ is a slowly varying linear operator. The solution $U$ can be a function of time if this problem corresponds to a system of ODEs, or it can be a function of space and time if this problem involves a system of PDEs. As we are only interested in the temporal behaviour of the solution $U$, we will only write $U=U(t)$. The initial condition for this problem is written as $U(0)=U_{i}$ and in the case where (A.1) is a PDE system, we suppose the boundary conditions can be written as $B(\tau) U=0$.

The asymptotic solution to this problem will first be derived using the method of multiple scales. An introductory discussion on this method can be found in Bender \& Orszag (1999) whereas an advanced treatment can be found in Kevorkian \& Cole (1996). The derivation presented here will be similar to two-step approach taken by Hennessy \& Münch (2013) where the solution $U$ is first projected into the eigenfunctions of $L$ and then the resulting system of ODEs is approximately solved using multiple 
scales. However, we are able to obtain a slightly more general result by not restricting our derivation to the top two eigenmodes, i.e., we consider all of the modes and not just the two corresponding to the eigenvalues with largest and second-largest real parts. Furthermore, we consider the case when the eigenvalues are complex.

To begin the derivation, we assume that the spectrum of $L$ (with possibly $B$ ) is discrete, that the eigenvalues are distinct for each value of $\tau$, and that the eigenfunctions form a complete set. Under these assumptions, we can write the solution $U$ as

$$
U(t)=\sum_{l=1}^{\infty} \alpha_{l}(t) v_{l}(\tau)
$$

where $v_{l}$ is a slowly varying eigenfunction given by

$$
L(\tau) v_{l}(\tau)=\lambda_{l}(\tau) v_{l}(\tau)
$$

and possibly $B(\tau) v_{l}=0$. Here $\lambda_{l}$ denotes the $l$-th eigenvalue and these are ordered according to the size of their real part. That is, we assume $\operatorname{Re}\left\{\lambda_{1}\right\}>\operatorname{Re}\left\{\lambda_{2}\right\}>\ldots>\operatorname{Re}\left\{\lambda_{n}\right\}$ as $n \rightarrow \infty$. We also define the adjoint eigenproblem as

$$
L^{*}(\tau) v_{l}^{*}(\tau)=\lambda_{l}^{\dagger}(\tau) v_{l}^{*}(\tau)
$$

where the dagger denotes complex conjugation, $L^{*}$ is the adjoint of the linear operator in (A.1), and $v_{l}^{*}$ denotes the adjoint eigenfunction. The eigenfunctions and their adjoints are normalised according to

$$
\left\langle v_{l}, v_{l}\right\rangle(\tau) \equiv 1, \quad\left\langle v_{k}^{*}, v_{l}\right\rangle(\tau) \equiv \delta_{k l},
$$

where $\delta_{k l}$ denotes the Kronecker delta and $\langle\cdot, \cdot\rangle$ denotes a possibly $\tau$-dependent inner product. We assume that the second argument in the inner product is complex conjugated in the operation.

By inserting the solution in (A.2) into (A.1) and taking the inner product with the adjoint eigenfunctions, we find that the functions $\alpha_{l}$ satisfy system of ODEs given by

$$
\frac{\mathrm{d} \alpha_{l}}{\mathrm{~d} t}-\left[\lambda_{l}(\tau)+\delta \gamma_{l l}(\tau)\right] \alpha_{l}(t)=\delta \sum_{j \neq l}^{\infty} \gamma_{l j}(\tau) \alpha_{j}(t), \quad l=1,2, \ldots
$$

where the $\gamma_{j l}$ are defined as

$$
\gamma_{j l}(\tau)=-\left\langle v_{j}^{*}, \partial_{\tau} v_{l}\right\rangle^{\dagger}(\tau)
$$

The initial conditions for this system are given by $\alpha_{l}(0)=\alpha_{l i}=\left\langle v_{l}^{*}, U_{i}\right\rangle^{\dagger}(0)$.

We now invoke the method of multiple scales to obtain an approximate solution to the ODE system in (A.6). However, the application of the method is not straightforward for this system because the time $t$ is not an appropriate time variable; see page 558 of Bender \& Orszag (1999) for an exemplary discussion about this. We therefore introduce several new times $T_{l}, l=1,2, \ldots$, that will replace $t$. These times are defined as

$$
T_{l}=\delta^{-1} \int_{0}^{\delta t}\left[\lambda_{l}(s)+\delta \gamma_{l l}(s)\right] \mathrm{d} s, \quad l=1,2, \ldots
$$


Using these new time variables, we write $\alpha_{l}(t)$ as $\alpha(\tau, T)$, where the argument $T$ without any subscript denotes dependence on each of the times $T_{l}$. By applying the chain rule, the ODE system in (A.6) can be written as

$$
\delta \frac{\partial \alpha_{l}}{\partial \tau}+\sum_{j=1}^{\infty}\left[\lambda_{j}(\tau)+\delta \gamma_{j j}(\tau)\right] \frac{\partial \alpha_{l}}{\partial T_{j}}-\left[\lambda_{l}(\tau)+\delta \gamma_{l l}(\tau)\right] \alpha_{l}=\delta \sum_{j \neq l}^{\infty} \gamma_{l j}(\tau) \alpha_{j}, \quad l=1,2, \ldots,
$$

where the initial condition now reads $\alpha_{l}(0,0)=\alpha_{l i}$. We now treat all of the time variables, namely $\tau$, $T_{1}, T_{2}, \ldots, T_{n}, n \rightarrow \infty$, as independent variables, and following Kevorkian and Cole (see Kevorkian \& Cole, 1996, pg. 395), we expand each $\alpha_{l}$ as an asymptotic series of the form

$$
\alpha_{l}(\tau, T)=\alpha_{l}^{(0)}\left(\tau, T_{l}\right)+\sum_{n=1}^{\infty} \delta^{n} \alpha_{l}^{(n)}(\tau, T)
$$

The initial conditions now read $\alpha_{l}^{(0)}(0,0)=\alpha_{l i}$ and $\alpha_{l}^{(n)}(0,0)=0$ for all $n$ and all $l$. We insert this expansion into the system (A.9), collect powers of $\delta$, and solve each problem sequentially.

The leading-order problem is given by

$$
\frac{\partial \alpha_{l}^{(0)}}{\partial T_{l}}-\alpha_{l}^{(0)}=0
$$

which has the solution

$$
\alpha_{l}^{(0)}\left(\tau, T_{l}\right)=P_{l}(\tau) e^{T_{l}}
$$

where $P_{l}(0)=\alpha_{l i}$. The determination of $P_{l}$ comes from eliminating secular terms in the $O(\delta)$ problem. This problem is given by

$$
\sum_{j=1}^{\infty} \lambda_{j} \frac{\partial \alpha_{l}^{(1)}}{\partial T_{j}}-\lambda_{l} \alpha_{l}^{(1)}=-\frac{\mathrm{d} P_{l}}{\mathrm{~d} \tau} e^{T_{l}}+\sum_{j \neq l}^{\infty} \gamma_{l j} P_{j} e^{T_{j}}
$$

where we have omitted the explicit dependence of the various functions on the slow time $\tau$ for notational convenience. We can treat this equation as a first order hyperbolic PDE and hence we can rewrite it in terms of its bicharacteristics. We therefore let $\mathrm{d} T_{i} / \mathrm{d} \xi=\lambda_{i}$ and write (A.13) as

$$
\frac{\partial \alpha_{l}^{(1)}}{\partial \xi}-\lambda_{l} \alpha_{1}^{(1)}=-\frac{\mathrm{d} P_{l}}{\mathrm{~d} \tau} e^{\lambda_{l} \xi}+\sum_{j \neq l}^{\infty} \gamma_{l j} P_{j} e^{\lambda_{j} \xi}
$$

where we make the remark that the $\xi$ derivative of $\alpha_{l}^{(1)}$ is only a partial derivative because this function still depends on $\tau$. From this new equation it is easy to see that any terms that are proportional to $\exp \left(\lambda_{l} \xi\right)$ will lead to secular terms in the solution for $\alpha_{l}^{(1)}$ and hence they need to be eliminated. This implies that functions $P_{l}$ are constants given by

$$
P_{l}(\tau) \equiv \alpha_{l i}
$$

The equation in (A.14) can be solved using an integrating factor and the solution, upon rewriting it in terms of the times $T_{l}$, is given by

$$
\alpha_{l}^{(1)}(\tau, T)=\sum_{j \neq l}^{\infty} \frac{\alpha_{j i} \gamma_{l j}(\tau)}{\lambda_{j}(\tau)-\lambda_{l}(\tau)} e^{T_{j}}+Q_{l}(\tau) e^{T_{l}}
$$


The functions $Q_{l}$ have initial conditions given by

$$
Q_{l}(0)=-\sum_{j \neq l}^{\infty} \frac{\alpha_{j i} \gamma_{l j}(0)}{\lambda_{j}(0)-\lambda_{l}(0)},
$$

and they are determined by eliminating the secular terms that otherwise occur in the $O\left(\delta^{2}\right)$ problem. This problem is given by

$$
\sum_{j=1}^{\infty} \lambda_{j} \frac{\partial \alpha_{l}^{(2)}}{\partial T_{j}}-\lambda_{l} \alpha_{l}^{(2)}=-\frac{\partial \alpha_{l}^{(1)}}{\partial \tau}-\gamma_{l l} \alpha_{l}^{(1)}+\sum_{j \neq l}^{\infty} \gamma_{l j} \alpha_{j}^{(1)}+\sum_{j=1}^{\infty} \gamma_{j j} \frac{\partial \alpha_{l}^{(1)}}{\partial T_{j}}
$$

Using the same approach as before, the terms that lead to secularity in the solution for $\alpha_{l}^{(2)}$ can be identified as the terms that are proportional to $\exp \left(\lambda_{l} \xi\right)$ once (A.16) is inserted into this equation and it is written in terms of its bicharacteristics. The elimination of these terms yields the following differential equation for $Q_{l}$ :

$$
\frac{\mathrm{d} Q_{l}}{\mathrm{~d} \tau}=\alpha_{l i} \sum_{j \neq l}^{\infty} \frac{\gamma_{l j}(\tau) \gamma_{j l}(\tau)}{\lambda_{l}(\tau)-\lambda_{j}(\tau)}
$$

which can be solved to obtain

$$
Q_{l}(\tau)=\sum_{j \neq l}^{\infty}\left[\alpha_{l i} \int_{0}^{\tau} \frac{\gamma_{l j}(s) \gamma_{j l}(s)}{\lambda_{l}(s)-\lambda_{j}(s)} \mathrm{d} s-\frac{\alpha_{j i} \gamma_{l j}(0)}{\lambda_{j}(0)-\lambda_{l}(0)}\right] .
$$

Therefore, the multiple-scales solution for the $\alpha_{l}$ can be written as

$$
\begin{aligned}
\alpha_{l}(t)=\alpha_{l i} e^{T_{l}} & +\delta \sum_{j \neq l}^{\infty} \frac{\alpha_{j i} \gamma_{l j}(\tau)}{\lambda_{j}(\tau)-\lambda_{l}(\tau)} e^{T_{j}} \\
& +\delta \sum_{j \neq l}^{\infty}\left[\alpha_{l i} \int_{0}^{\tau} \frac{\gamma_{l j}(s) \gamma_{j l}(s)}{\lambda_{l}(s)-\lambda_{j}(s)} \mathrm{d} s-\frac{\alpha_{j i} \gamma_{l j}(0)}{\lambda_{j}(0)-\lambda_{l}(0)}\right] e^{T_{l}}+O\left(\delta^{2}\right),
\end{aligned}
$$

where $\tau=\delta t$ and the $T_{l}$ are given in (A.8). When this solution is combined with the eigenfunction expansion in (A.2), we effectively have a multiple-scales approximation to the original linear problem in (A.1).

\section{A.1 An alternative derivation using the WKB method}

The asymptotic solution presented above can also be derived using the WKB approximation (Bender \& Orszag, 1999). This may be a more useful approach when trying to resolve the situation when eigenvalues momentarily become identical; however, we do not address this issue here. We start by considering the slowly varying linear problem of the form

$$
\frac{\partial U}{\partial t}=L(\delta t) U
$$

with $\delta \ll 1$, subject to the initial condition $U(0)=U_{i}$ and possibly with slowly-varying boundary conditions given by $B(\tau) U=0$. We now write the problem exclusively in terms of the slow time $\tau$ by making 
the change of variable $t=\delta^{-1} \tau$, thus giving

$$
\delta \frac{\partial U}{\partial \tau}=L(\tau) U
$$

We suppose that the solution $U$ can be written in the form of the usual WKB ansatz, namely

$$
U(\tau)=\Phi(\tau) e^{\phi(\tau) / \delta}
$$

where it is assumed that $\phi$ depends only on $\tau$ and satisfies the initial condition $\phi(0)=0$. The function $\Phi$ can depend on other independent variables such as space, but we do not explicitly write this dependence. By inserting this solution into (A.23) the problem becomes

$$
\delta \frac{\partial \Phi}{\partial \tau}+\frac{\mathrm{d} \phi}{\mathrm{d} \tau} \Phi=L \Phi
$$

The functions $\Phi$ and $\phi$ are expanded as asymptotic series of the form

$$
\begin{aligned}
& \Phi(\tau)=\Phi^{(0)}(\tau)+\delta \Phi^{(1)}(\tau)+\delta^{2} \Phi^{(2)}(\tau)+O\left(\delta^{3}\right) \\
& \phi(\tau)=\phi^{(0)}(\tau)+\delta \phi^{(1)}(\tau)+\delta^{2} \phi^{(2)}(\tau)+O\left(\delta^{3}\right)
\end{aligned}
$$

where $\phi^{(n)}(0)=0$ for all values of $n$, and by collecting powers of $\delta$ a sequence of sub-problems can be formulated.

The $O(1)$ problem is given by

$$
\frac{\mathrm{d} \phi^{(0)}}{\mathrm{d} \tau} \Phi^{(0)}=L \Phi^{(0)},
$$

and it follows that $\Phi^{(0)}$ is an eigenfunction of $L$ and $\partial_{\tau} \phi^{(0)}$ is equal to the corresponding eigenvalue. We let

$$
\Phi^{(0)}(\tau)=\alpha_{l i} v_{l}(\tau), \quad \phi^{(0)}(\tau)=\int_{0}^{\tau} \lambda_{l}(s) \mathrm{d} s
$$

where $v_{l}$ is the $l$-th eigenfunction of $L$ that satisfies $\left\langle v_{l}, v_{l}\right\rangle=1, \lambda_{l}$ is the corresponding eigenfunction, and $\alpha_{l i}$ is a normalisation factor.

The leading-order solution for $U$ is obtained by summing over all of the eigenmodes to give

$$
U(\tau)=\sum_{l=1}^{\infty} \alpha_{l i} \exp \left[\int_{0}^{\tau} \lambda_{l}(s) \mathrm{d} s\right] v_{l}(\tau)+O(\delta) .
$$

By imposing the initial condition and taking the inner product with the (normalised) adjoint eigenfunctions it follows that

$$
\alpha_{l i}=\left\langle v_{l}^{*}, U_{i}\right\rangle^{\dagger}(0)
$$

Here we have assumed that the adjoint eigenfunctions are normalised according to $\left\langle v_{j}^{*}, v_{k}\right\rangle=\delta_{j k}$, where $\delta_{j k}$ is the Kronecker delta.

Proceeding further, the $O(\delta)$ problem is given by

$$
\frac{\partial \Phi^{(0)}}{\partial \tau}+\frac{\mathrm{d} \phi^{(1)}}{\mathrm{d} \tau} \Phi^{(0)}+\frac{\mathrm{d} \phi^{(0)}}{\mathrm{d} \tau} \Phi^{(1)}=L \Phi^{(1)} .
$$


By making use of (A.28) this problem can be written as

$$
\left(L-\lambda_{l} I\right) \Phi^{(1)}=\frac{\partial v_{l}}{\partial \tau}+\frac{\mathrm{d} \phi^{(1)}}{\mathrm{d} \tau} v_{l},
$$

where $I$ is the identity operator. The operator on the left-hand side of this equation is clearly singular; thus, for a solution to exist the Fredholm solvability condition must be satisfied. Imposing this condition provides an equation for $\phi^{(1)}$. More specifically, we take the inner product of the right-hand side of (A.32) with $v_{l}^{*}$ and we find that

$$
\phi^{(1)}(\tau)=\int_{0}^{\tau} \gamma_{l l}(s) \mathrm{d} s
$$

where $\gamma_{j k}(\tau)=-\left\langle v_{j}^{*}, \partial_{\tau} v_{k}\right\rangle^{\dagger}(\tau)$ for all $j$ and $k$.

The solution for $\Phi^{(1)}$ can be found via an eigenfunction expansion. We let

$$
\Phi^{(1)}(\tau)=\alpha_{l i} \sum_{j=1}^{\infty} a_{j}(\tau) v_{j}(\tau)
$$

and by inserting this into (A.32), the coefficient functions are straightforward to obtain. In particular, we find

$$
a_{j}(\tau)=-\frac{\gamma_{j l}(\tau)}{\lambda_{j}(\tau)-\lambda_{l}(\tau)}, \quad j \neq l
$$

The function $a_{l}(\tau)$ cannot be determined from this problem, so we proceed to the next order.

The $O\left(\delta^{2}\right)$ problem is given by

$$
\left(L-\lambda_{l} I\right) \Phi^{(2)}=\frac{\partial \Phi^{(1)}}{\partial \tau}+\frac{\mathrm{d} \phi^{(2)}}{\mathrm{d} \tau} \Phi^{(0)}+\frac{\mathrm{d} \phi^{(1)}}{\mathrm{d} \tau} \Phi^{(1)},
$$

and for this equation to admit a solution the right-hand side must satisfy a similar Fredholm solvability condition. Taking the inner product of the right-hand side of (A.36) with $v_{l}^{*}$ yields a differential equation for $\phi^{(2)}$ which can be solved to obtain

$$
\phi^{(2)}(\tau)=a_{l}(0)-a_{l}(\tau)-\sum_{j \neq l} \int_{0}^{\tau} \frac{\gamma_{j l}(s) \gamma_{l j}(s)}{\lambda_{j}(s)-\lambda_{l}(s)} \mathrm{d} s .
$$

To proceed further, we insert the $O(1)$ and $O(\delta)$ solutions along with (A.37) into the WKB ansatz (A.24) and we expand the exponential as

$$
\exp \left(\delta^{-1} \phi^{(0)}+\phi^{(1)}+\delta \phi^{(2)}+O\left(\delta^{2}\right)\right)=\left(1+\delta \phi^{(2)}\right) \exp \left(\delta^{-1} \phi^{(0)}+\phi^{(1)}\right)+O\left(\delta^{2}\right) .
$$

This allows the unknown function $a_{l}(\tau)$ to be eliminated from the problem. However, its initial value $a_{l}(0)$ still appears, and this is chosen below to satisfy the initial condition.

The solution $U$ is obtained by summing over all of the modes to obtain

$$
\begin{aligned}
U(\tau)=\sum_{l=1}^{\infty} \alpha_{l i}\left[\left(1+\delta a_{l}(0)\right) v_{l}(\tau)\right. & +\delta \sum_{j \neq l}^{\infty} \frac{\gamma_{j l}(\tau)}{\lambda_{l}(\tau)-\lambda_{j}(\tau)} v_{j}(\tau) \\
& \left.+\left(\sum_{j \neq l}^{\infty} \int_{0}^{\tau} \frac{\gamma_{l j}(s) \gamma_{j l}(s)}{\lambda_{l}(s)-\lambda_{j}(s)} \mathrm{d} s\right) v_{l}(\tau)\right] e^{T_{l}(\tau)}+O\left(\delta^{2}\right),
\end{aligned}
$$


where $T_{l}$ is defined as

$$
T_{l}(\tau)=\delta^{-1} \int_{0}^{\tau}\left[\lambda_{l}(s)+\delta \gamma_{l l}(s)\right] \mathrm{d} s .
$$

To simplify this solution we write it is an eigenfunction expansion of the form

$$
U(\tau)=\sum_{l=1}^{\infty} \alpha_{l}(\tau) v_{l}(\tau)
$$

where $\alpha_{l}(0)=\alpha_{l i}$ for all values of $l$. Using the fact that

$$
\sum_{l=1}^{\infty} \alpha_{l i}\left(\sum_{j \neq l} \frac{\gamma_{j l}}{\lambda_{l}-\lambda_{j}} v_{j}\right) e^{T_{l}}=\sum_{j=1}^{\infty}\left(\sum_{l \neq j}^{\infty} \frac{\alpha_{l i} \gamma_{j l}}{\lambda_{l}-\lambda_{j}} e^{T_{l}}\right) v_{j},
$$

we find that the functions $\alpha_{l}$ are given by

$$
\begin{aligned}
\alpha_{l}(\tau)=\alpha_{l i}\left(1+\delta a_{l}(0)\right) e^{T_{l}(\tau)} & +\delta \sum_{j \neq l}^{\infty} \frac{\alpha_{j i} \gamma_{l j}(\tau)}{\lambda_{j}(\tau)-\lambda_{l}(\tau)} e^{T_{j}(\tau)} \\
& +\delta\left(\sum_{j \neq l}^{\infty} \alpha_{l i} \int_{0}^{\tau} \frac{\gamma_{j l}(\tau) \gamma_{l j}(\tau)}{\lambda_{l}(\tau)-\lambda_{j}(\tau)} \mathrm{d} s\right) e^{T_{l}(\tau)}+O\left(\delta^{2}\right) .
\end{aligned}
$$

By imposing the initial condition $\alpha_{l}(0)=\alpha_{l i}$ we can solve for $a_{l}(0)$, thus yielding

$$
\begin{aligned}
\alpha_{l}(\tau)=\alpha_{l i} e^{T_{l}(\tau)} & +\delta \sum_{j \neq l}^{\infty} \frac{\alpha_{j i} \gamma_{l j}(\tau)}{\lambda_{j}(\tau)-\lambda_{l}(\tau)} e^{T_{j}(\tau)} \\
& +\delta\left(\sum_{j \neq l}^{\infty} \alpha_{l i} \int_{0}^{\tau} \frac{\gamma_{j l}(\tau) \gamma_{l j}(\tau)}{\lambda_{l}(\tau)-\lambda_{j}(\tau)} \mathrm{d} s-\frac{\alpha_{j i} \gamma_{l j}(0)}{\lambda_{j}(0)-\lambda_{l}(0)}\right) e^{T_{l}(\tau)}+O\left(\delta^{2}\right),
\end{aligned}
$$

which is equivalent to the expression obtained using the method of multiple scales. 